\title{
ANÁLISIS DEL DISEÑO \\ DE UNA RED INTEGRADA DE SERVICIOS DE SALUD*
}

\author{
Recibido: 20 de diciembre de 2015 • Aprobado: 06 de julio de 2016 \\ DOI: $10.22395 /$ seec.v19n40a7 \\ Dora Marcela Pulgarín Henao* \\ Raúl Andrés Tabarquino Muñoz
}

\section{RESUMEN}

Este artículo presenta el análisis de los beneficios del diseño de una red integrada de servicios de salud basada en la gestión de la cadena de suministro; para ello se parte del estudio de investigación realizado en una Empresa Social del Estado ESE Oriente, de la ciudad de Cali (Colombia), en razón a su riqueza metodológica sustentada en el análisis cualitativo y cuantitativo a partir de los referentes teóricos y conceptuales de los atributos mínimos de una red integrada de servicios de salud y el scor model. La principal conclusión del análisis es que las redes integradas de servicios de salud responden a procesos de gestión y planeación en la entrega de servicios de salud de manera preventiva y curativa, que implican necesariamente interrelación y coordinación con características de calidad, personal, recursos, atención e infraestructura.

\section{PALABRAS CLAVE}

Construcción de redes, cadena de suministro, estructura organizacional, servicios de salud y redes.

\section{CLASIFICACIÓN JEL}

H75, L14, M10

\section{CONTENIDO}

Introducción; 1. Referentes Teóricos y Conceptuales; 2. Metodología; 3. Resultados y Discusión; 4. Conclusiones; Bibliografía, Anexos.

* El presente artículo de investigación es producto de los avances de la tesis doctoral del profesor Tabarquino en cuanto a la aplicación de la metodología de redes, y los resultados de investigación de la tesis titulada "Diseño de una red integrada de servicios de salud basada en la gestión de la cadena de suministro: caso de tres organizaciones de salud de la ciudad de Santiago De Cali", desarrollada por la investigadora Pulgarín bajo la tutoría del profesor Arley Torres entre los años 2013-2015, en el programa de Maestría en logística integral de la Universidad Autónoma de Occidente, Cali, Colombia.

** Profesional en Finanzas y Negocios internacionales, Universidad Santiago de Cali, Cali, Colombia. Magíster en Logística Integral, Universidad Autónoma de Occidente de Cali, Cali, Colombia. Coordinadora de Comercio Exterior, Agencia Aduanera Servadi, Cali, Colombia. Docente de Práctica Empresarial. Dirección: Cr 25 a \# 27-92., Cali, Colombia. Correo electrónico: mpulgarin@hotmail.com.

*** Economista, Universidad del Valle, Cali, Colombia. Magíster en Políticas Públicas, Universidad del Valle, Cali, Colombia. Candidato a PhD en Administración, Universidad del Valle, Cali, Colombia. Investigador del Grupo Gestión y Políticas Públicas, clasificado en Categoría B de Colciencias, Universidad del Valle, Cali, Colombia. Docente tiempo completo, Universidad del Valle, Cali, Colombia. Dirección: Cl 4b \#36b36, Edificio 124, Departamento de Administración y Organizaciones. Cali, Colombia. Correo electrónico: ranta0831@gmail.co; raul.tabarquino@correounivalle.edu.co. 


\section{INTEGRATED NETWORK FOR HEALTH SERVICES ANALYSIS ABSTRACT}

This article analyses the benefits of designing an integrated network for health services based on supply chain management. For this purpose, the study used a previous study carried out by a Government Company called ESE Oriente, from Cali city, given its methodological content based on qualitative and quantitative analysis parting from theoretical and conceptual referents of the minimum attributes of an integrated service network for health service, which respond to management and planning processes in the delivery of health services for prevention and healing, which necessarily imply the interrelation and coordination with personal quality, resources, attention and infrastructure characteristics.

\section{KEY WORDS}

Network building, supply chain, organizational structure, heath services and networks.

\section{JEL CLASSIFICATION}

H75, L14, M10

\section{CONTENT}

Introduction; 1. Theoretical and conceptual referents; 2. Methodology; 3. Results and discussions; 4. Conclusions; Bibliography, Attachments.

\section{ANALISE DO DESENHO DE UMA REDE INTEGRADA DE SERVIÇOS DE SAÚDE RESUMO}

Este artigo apresenta a análise dos benefícios do desenho de uma rede integrada de serviços de saúde baseada na gestão da cadeia de subministro, para isto seja parte do estudo de investigação realizado em una Empresa Social do Estado ESE Oriente, da cidade de Cali (Colômbia), em razão a sua riqueza metodológica sustentada na análise qualitativo e quantitativo a partir dos referentes teóricos e conceituais dos atributos mínimos de uma rede integrada de serviços de saúde e o scor model. A principal conclusão da análise é que as redes integradas de serviços de saúde, respondem a processos de gestão e planejamento na entrega de serviços de saúde de maneira preventiva e curativa, que implicam necessariamente inter-relação e coordenação com características de qualidade, pessoal, recursos, atenção e infraestrutura.

\section{PALAVRAS CHAVE}

Construção de redes, Cadeia de subministro, Estrutura organizacional, Serviços de saúde e redes.

\section{CLASSIFICAÇÃO JEL} H75, L14, M10

\section{CONTEÚDO}

Introdução; 1. Referentes Teóricos e Conceituais; 2. Metodologia; 3. Resultados e Discussão; 4. Conclusões; Bibliografia, Anexos. 


\section{INTRODUCCION}

América Latina y el Caribe presentan escenarios de necesidades básicas insatisfechas entre las cuales se encuentra el acceso a servicios públicos domiciliarios como el agua potable, la energía y el alcantarillado, lo que ratifica la precariedad de la población ubicada en escalas menos favorecidas y, como consecuencia directa, el aumento de la demanda de servicios en salud, según estudios de la OPS (2010) y Artaza y otros (2011). Para el caso colombiano, Pulgarín (2015) señala que el modelo de gestión actual en salud agudiza la precariedad, debido a que las organizaciones realizan, de manera directa e indirecta, la coordinación entre agentes y recursos; además, las entidades en salud (hospitales, centros de salud, puestos de salud, clínicas) no trabajaban en red, es decir, que sus estructuras no cuentan con capacidad técnica de reacción, absorción de insumos y flujo de capital humano.

El MSPC (2016, p. 3), con Resolución 429, adopta la política de atención integral en salud (PAIS), en la cual, propone la implementación de las Redes Integradas de Servicios de Salud (RISS): artículo 5 "... conjunto articulado de prestadores de servicios de salud u organizaciones funcionales de servicios de salud, públicos y privados; ubicados en un ámbito territorial definido de acuerdo con las condiciones de operación....".

Por lo anterior, este artículo tiene como objetivo principal analizar, a la luz del enfoque de redes, cuáles beneficios traería el diseño de una red integrada de servicios de salud, basada en la gestión de la cadena de suministro. Se parte del estudio realizado en la Empresa Social del Estado -ESE Oriente ${ }^{1}$ - de la ciudad de Cali, que toma como punto de partida los atributos de una red integrada de servicios de salud establecidos por la Organización Panamericana de la Salud (OPS) y las herramientas del scor model; para el caso de la dinámica en red se utiliza el análisis de redes con el fin de precisar, visualizar y analizar la red de la cadena de suministro de la ESE Oriente antes y después de aplicar el concepto de RISS y cadena de suministro.

\section{REFERENTES TEÓRICOS Y CONCEPTUALES}

\subsection{Las redes integradas de servicios de salud}

Las redes integradas de servicios de salud se definen como un conjunto de organizaciones y actores que permanentemente ordenan, coordinan y promueven programas y servicios a una población. La anterior definición se basa en autores como Vásquez y Vargas (2006), Lau y Soler (2009), OPS (2010), Artaza, Méndez, Holder y Suárez (2011), Morales (2011), Barragán, Riaño y Martínez (2012), Balladelli (2013) y Ocampo-Rodríguez y otros (2013). 
Las redes integradas de servicios de salud, partiendo de autores como Kongstvedt y Gates (1996), Gillies , Shortell y Anderson (1993), OPS, (2010), Satinsky (1998), Lin, Lin y Lin (2010), Barragán, Riaño y Martínez (2012) y Pike y Mongan (2014), se clasifican de acuerdo con tres categorías: a) red de personal de salud, b) red de personal y establecimientos de salud; y c) red de personal, establecimientos y aseguradoras de salud.

\subsubsection{Los atributos esenciales de las RISS}

Independiente del modelo organizacional de RISS que se aplique por los diferentes contextos de cada región, se deben conservar atributos mínimos establecidos por la organización Panamericana en Salud (OPS) para su correcto funcionamiento. A continuación, se describen cada uno de ellos:

Tabla 1. Atributos mínimos de una RISS

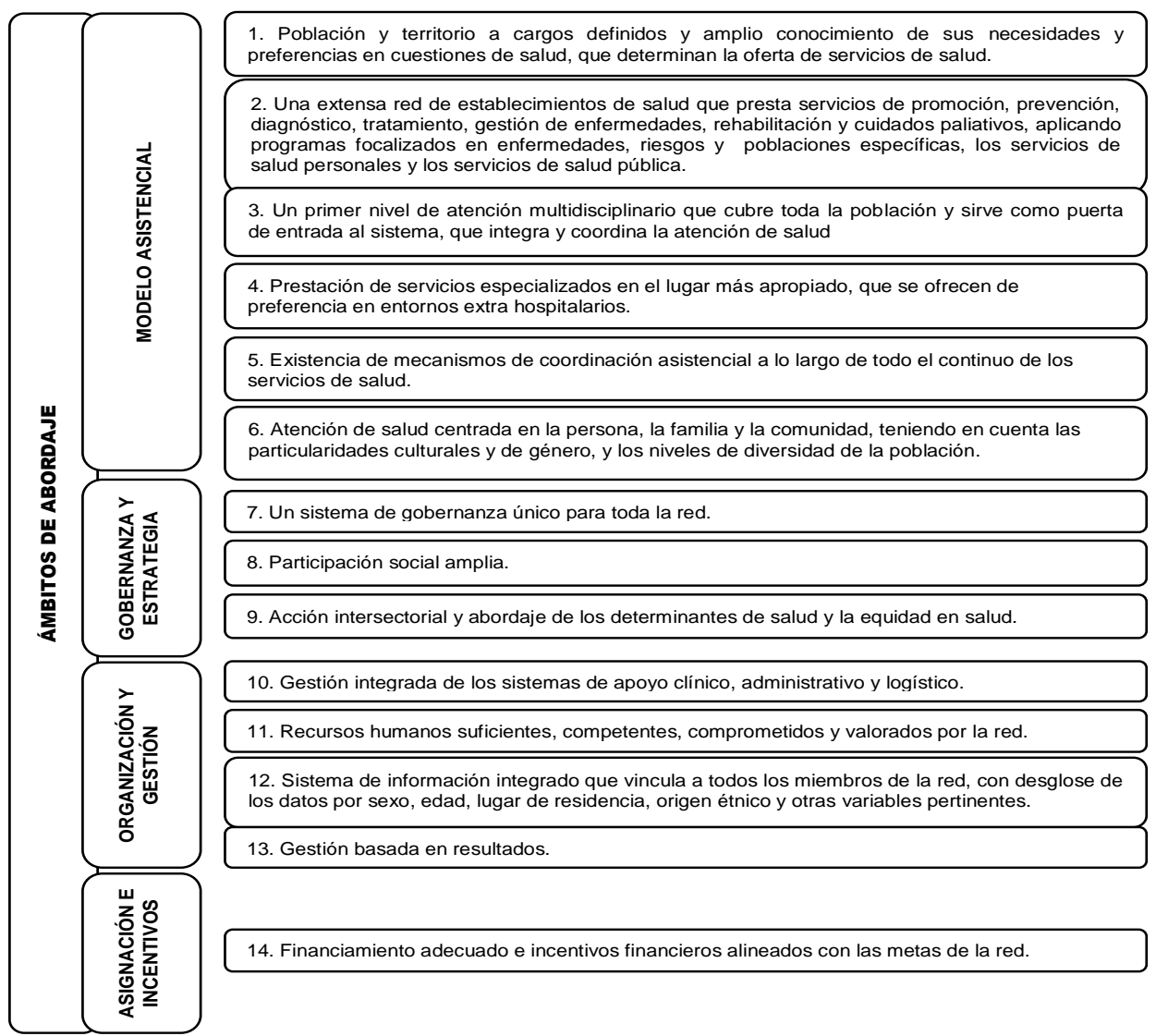

Fuente: Pulgarín (2015, p. 31) 


\subsection{Cadena de suministro}

La cadena de suministro se define como una la red de organizaciones conectadas e interdependientes entre sí, que genera cooperación para el control, manejo y mejoramiento continuo del flujo de recursos, materiales e información desde los proveedores hasta los usuarios finales, según Jiménez (2004), Capó, Tomás y Expósito-Langa (2007), Franco, Flórez y Ochoa (2008) y Hernández-Rodríguez (2012).

La cadena de suministro es un componente transversal, puesto que la conforman todos los procesos involucrados, de manera directa o indirecta, con la satisfacción las necesidades del cliente, como pueden ser los procesos de manufactura, distribución, ventas y servicio al cliente intergrados en un solo negocio para garantizar la calidad y velocidad en la satisfacción del consumidor como lo plantean Vachon y Klassen (2002), Jiménez y Hernández (2002), Cambra y Polo (2008) y Suárez y otros (2015).

\subsubsection{El modelo Supply Chain Operations Reference model (SCOR)}

El modelo SCOR estandariza la terminología y los procesos de una cadena de suministro para comparar y analizar diferentes alternativas y estrategias para la cadena en general o para cada uno de sus integrantes; no tiene en cuenta modelos matemáticos ni heurísticos; se organiza en torno a los cinco procesos principales de gestión: planear, procurar, transformar, entregar y devolver, como se ilustra en la figura 1.

Figura 1. Modelo SCOR y su organización por procesos

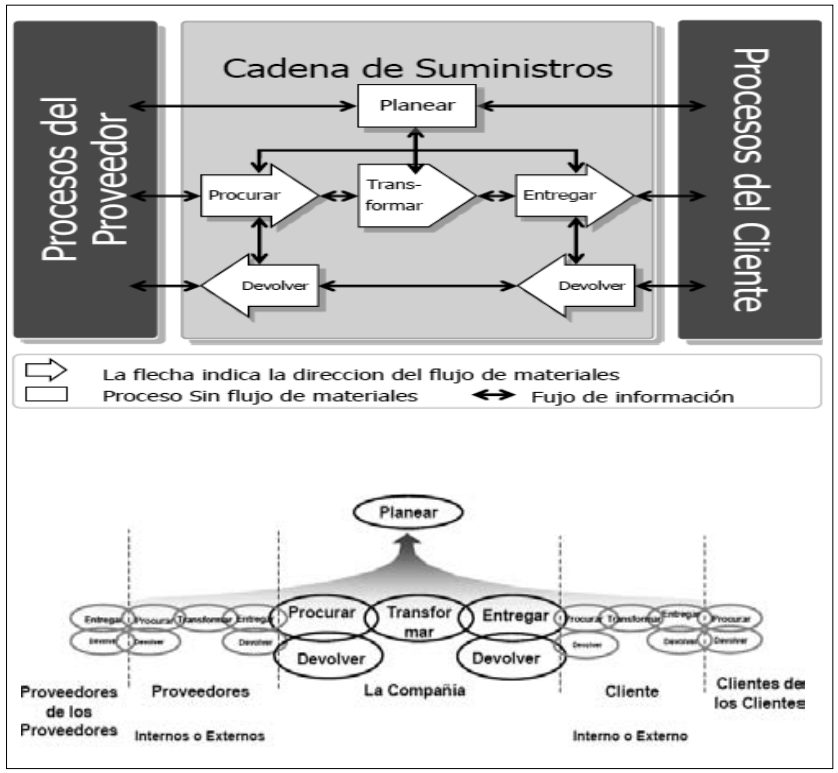

Fuente: Pulgarín (2015, p. 34) 
El modelo SCOR ofrece una visión clara de los procesos desarrollados en la cadena de suministros, lo que permite optimizar el uso de recursos y facilitar los procesos de arbitraje, tal y como lo plantean Brennan (1998), Vickery y otros (2003), Breen (2004), Beery y Vallianatos (2004), Stadtler y Kilger (2005), Mckone , Hamilton y Willis (2005), García y otros (2009), Sanchis, Poler y Ortiz (2009), Amaya y otros (2010), Reveco y Weber (2011), Aguilar, Garrido y Gallego (2013) y Aguilar y Garrido (2013).

Según Pulgarín (2015, p.35), el modelo SCOR abarca todas las interacciones: i) Los clientes, desde la entrada de órdenes hasta el pago de las facturas; ii) Las transacciones físicas de materiales, desde los proveedores de los proveedores (Suppliers), hasta los clientes de los clientes (Customers), incluyendo equipos, suministros, repuestos, productos a granel, software, etc.; iii) Las interacciones con el mercado, desde la demanda agregada hasta el cumplimiento de cada orden. Sin embargo, no intenta describir cada proceso de negocio o actividad; y iv) Específicamente el modelo SCOR no contiene ventas ni marketing (generación de la demanda); desarrollo del producto; investigación y desarrollo ni algunos elementos de servicio posventa al cliente. El modelo SCOR no abarca -pero presupone la existencia de- las actividades de recursos humanos, capacitación, sistemas, administración, así como las del aseguramiento de la calidad, entre otras.

\section{METODOLOGÍA}

La metodología utilizada se basa en la propuesta por Eisenhardt (1989) y Patton (2002). Las fuentes cualitativas de información son la revisión de literatura en RISS, cadena de suministro y Scor Model; el análisis documental a la información suministrada por la ESE Oriente en relación con los programas y actividades del Plan de Desarrollo Institucional 2012-2015; el análisis de entrevistas al exgerente de la ESE Norte de Cali y al gerente de la ESE Oriente de Cali formuladas a partir del scor model; y los atributos mínimos de una RISS establecidos por la OPS (2010).

Las fuentes de información cuantitativas son bases de datos 2012 al 2014 obtenidas de la Secretaria de Salud Municipal de Cali y la Alcaldía de Santiago de Cali, con información de las comunas 13, 14, 15 y 21 sobre población, morbilidad, mortalidad, partos e histórico de pacientes atendidos. Además, se tuvo acceso a las auditorías a la Red de Salud Oriente ESE 2102-2013. Por parte de la ESE Oriente se obtuvo las bases de datos con información de la población atendida, capacidad instalada y estado de resultados 2012 a 2014.

El periodo de realización del trabajo de campo y del proceso investigativo comprendió los meses de julio a noviembre de 2014; para la elección de las personas y 
grupos del trabajo de campo, se tuvieron en cuenta las funciones que desempeñan en la Ese Oriente; se procesaron la información y los indicadores en cuanto a las siguientes fases: i) Descripción del estado de la cadena de suministro de la ESE Oriente de Santiago de Cali, aplicando el modelo SCOR, en relación a los aspectos técnicos, administrativos, legales y financieros, con el fin de identificar actores claves en la red y estandarizar los procesos para que respondan a la dinámica de una RISS; ii) Evaluación de los atributos mínimos de una RISS en la ESE Oriente; iii) Diseño de la RISS, basada en la gestión integral de la cadena de suministro como plataforma técnica para asegurar el desarrollo y fortalecimiento institucional e interinstitucional.

\section{RESULTADOS Y DISCUSIÓN}

\subsection{Descripción del estado de la cadena de suministro de la ESE Oriente de Santiago de Cali con la aplicación del modelo SCOR}

Al aplicar el modelo SCOR a la cadena de suministro de la ESE Oriente de Cali, se delimitan dos grandes ejes de la logística hospitalaria que tienen que ver con el flujo de materiales, según Pulgarín (2015, p. 56): i) El abastecimiento, que incluye la compra, la recepción y la gestión de inventarios, y ii) La distribución o reabastecimiento, que hace el seguimiento de los diferentes productos desde las zonas de almacenamiento hasta los diferentes puntos de utilización.

Figura 2. Mapa del proceso de planeación de abastecimiento

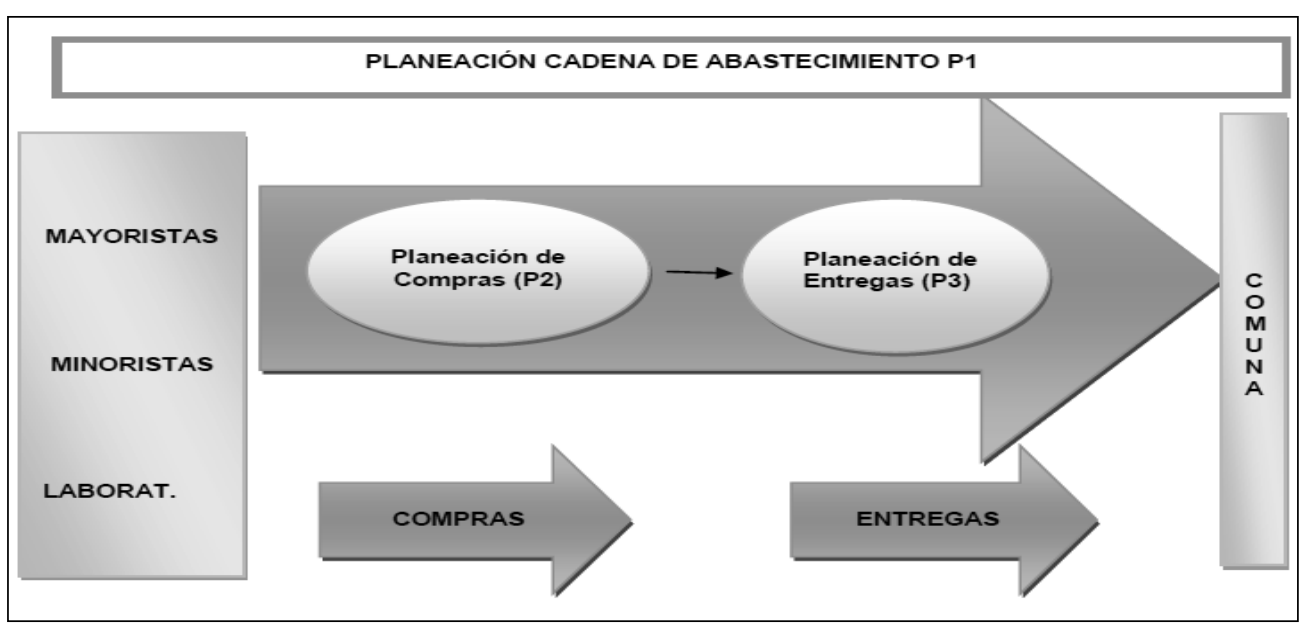

Fuente: Pulgarín (2015, p. 57) 
Pulgarín (2015, p. 57) evidenció que la ESE Oriente no trabaja con pronósticos de demanda (patologías-número de usuarios) para programar su cadena de suministro; por lo tanto, el punto de partida de la cadena de suministro de esta entidad se da en la planeación de compras, P2:

A. Planeación de compras P2. La ESE Oriente efectúa en primera instancia un presupuesto anual de compras a partir del histórico de consumo. En el transcurso del año las compras se realizan de manera trimestral, alimentadas con las órdenes de compras de cada una de las entidades que conforman la red, las cuales son ordenadas y consolidadas por el almacén de abastecimiento del Hospital Carlos Holmes Trujillo. La orden de compra consolidada se distribuye entre diferentes proveedores tanto mayoristas como minoristas.

B. Planeación de entregas (P3). Dentro de este punto de la cadena es importante destacar que la ESE Oriente maneja la distribución a sus 24 puntos de servicio con vehículos propios acondicionados para el transporte de pasajeros, cuya función principal es servir a todos los requerimientos de la ESE Oriente, y como función auxiliar sirven en su momento para la distribución de bienes. Por otro lado, el Hospital Carlos Holmes Trujillo se encarga también de abastecer un almacén más pequeño que funciona dentro del mismo edificio; se puede decir, grosso modo, que funciona como un sistema de abastecimiento conocido como el doble cajón o procedimiento de Kanban que es definido por Amaya y otros (2010) como un sistema sencillo de inventario para insumos de poco valor.

\subsection{Evaluación de los 14 atributos de la RISS para la ESE Oriente de Cali}

El criterio para realizar el análisis de red de la ESE Oriente en la ciudad de Cali, se basa en el concepto de la cadena de suministro propuesta por Starfield (1992,1998 y 2009), Giannakis, Croom y Slack (2004), Javalgi y Reinsenwithz (2001), Toro (2008), Montenegro y otros (2011), porque cumple con dos características: la primera, ser plataforma para el desarrollo de una RISS, y la segunda, por su relación directa con la prestación del servicio (la satisfacción del buen servicio al usuario). Se evaluaron los 14 atributos mínimos (OPS, 2010) de la RISS para la ESE Oriente dentro de su funcionamiento administrativo y operativo; el resultado de la evaluación en términos numéricos y porcentuales arroja como conclusión determinante que la ESE Oriente responde en su mayoría a una red parcialmente integrada (57,14 \%), y solo cumple con un atributo de RISS que equivale al 7,14\% 
Análisis del diseño de una red integrada de servicios de salud

Tabla 3. Evaluación de los 14 atributos mínimos de una RISS para la ESE Oriente

\begin{tabular}{|l|c|c|}
\hline Red & Atributos & Peso $\%$ \\
\hline Red fragmentada & $1,2,3,4,5,6,13$ y 14 & $35,71 \%$ \\
\hline Red parcialmente Integrada & $7,8,10,11$ y 12 & $57,14 \%$ \\
\hline Red Integrada -RISS- & 9 & $7,14 \%$ \\
\hline
\end{tabular}

Fuente: Elaboración propia a partir de Pulgarín (2015, p. 63-86)

\subsection{Diseño de la RISS para la Ese Oriente de Cali a partir de la cadena suministro}

\subsubsection{Relaciones entre los subsistemas de una IPS}

Las instituciones prestadoras de salud (IPS) también cuentan con diferentes plataformas las cuales son las encargadas de organizar las actividades misionales de la institución, entre ellas: i) Plataforma administrativa y financiera; ii) Plataforma gerencia, iii) Plataforma de distribución y iv) Plataforma comercial. Respecto a los proveedores se hace una clara diferencia entre los proveedores de bienes y los proveedores de servicios, puesto que la dinámica que se desarrolla con ellos difiere. En la figura 3 se visualiza la complejidad de las relaciones entre los diferentes subsistemas y agentes que hacen parte de las instituciones prestadoras de servicios de salud.

Figura 3. Relaciones entre los subsistemas de una IPS

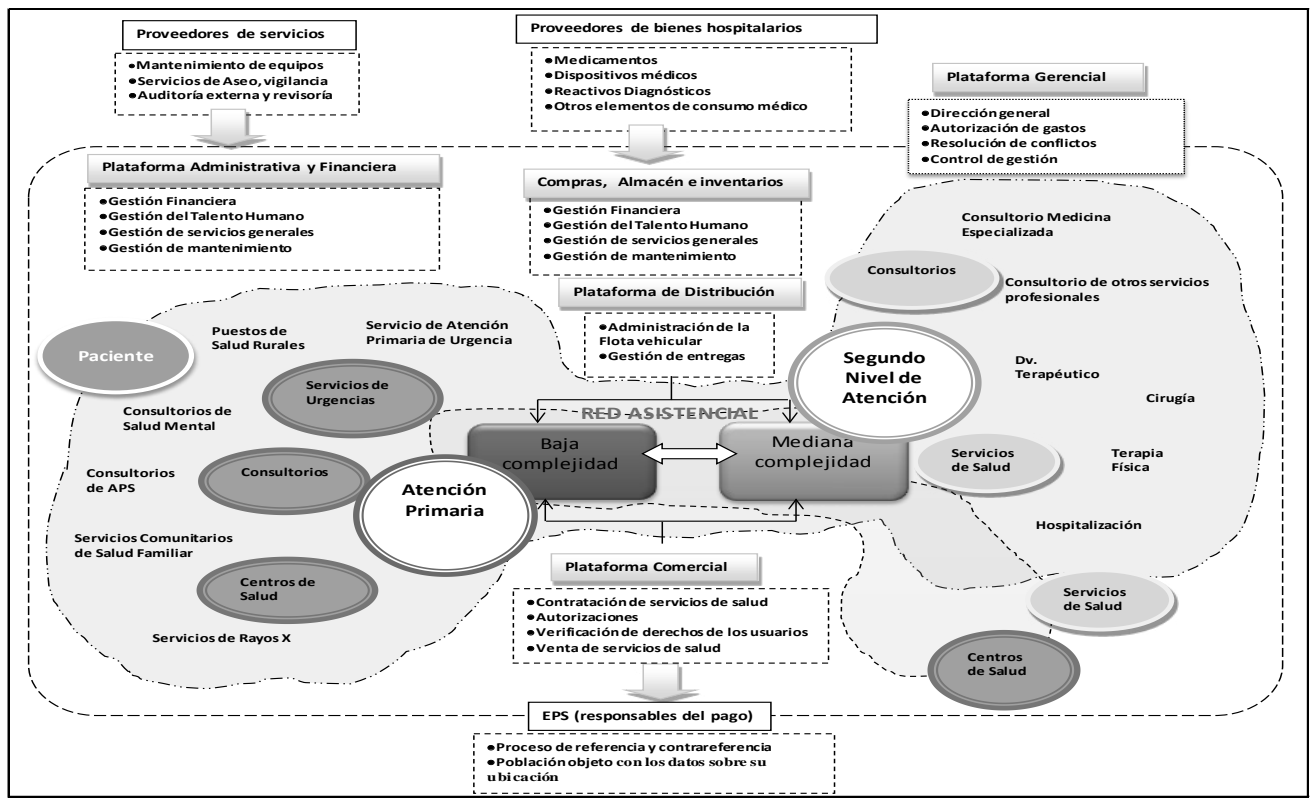

Fuente: Pulgarín $(2015$, p. 88) 


\subsubsection{Diseño de la RISS}

Pulgarín (2015) plantea a través de una base conceptual, el diseño de RISS con herramientas Logísticas/administrativas orientadas a promover la coordinación entre actores públicos y privados con miras a planificar, implementar y controlar el flujo de medios de transporte, el flujo de carga y el flujo de información de forma eficaz con el propósito de satisfacer los requerimientos de los pacientes (cumplir con los principios de la atención en salud).

Tabla 4. Diseño para la ESE Oriente para que funcione como una RISS

\begin{tabular}{|c|c|c|}
\hline $\begin{array}{l}\text { Ámbitos de } \\
\text { abordaje }\end{array}$ & Atributos OPS & $\begin{array}{c}\text { Herramientas Logísticas/ } \\
\text { administrativas }\end{array}$ \\
\hline \multirow{6}{*}{ 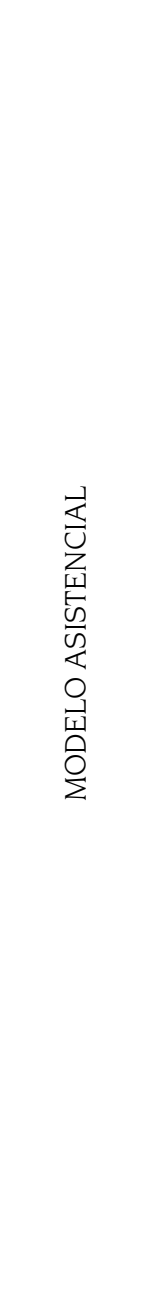 } & $\begin{array}{l}\text { 1. Población y territorio a cargo, definido y amplio } \\
\text { conocimiento de sus necesidades y preferencias } \\
\text { en cuestiones de salud, que determinan la oferta } \\
\text { de servicios de salud. }\end{array}$ & $\begin{array}{l}\text { - Sistema CRM - Cloud compu- } \\
\text { ting. }\end{array}$ \\
\hline & $\begin{array}{l}\text { 2. Una extensa red de establecimientos de salud } \\
\text { que presta servicios de promoción, prevención, } \\
\text { diagnóstico, tratamiento, gestión de enfermeda- } \\
\text { des, rehabilitación y cuidados paliativos, y que } \\
\text { integra los programas focalizados en enferme- } \\
\text { dades, riesgos y poblaciones específicas, los } \\
\text { servicios de salud personales y los servicios de } \\
\text { salud pública. }\end{array}$ & $\begin{array}{l}\text { - Implementación sistema } \\
\text { CRM - Segmentación }\end{array}$ \\
\hline & $\begin{array}{l}\text { 3. Un primer nivel de atención multidisciplinario } \\
\text { que abarca a toda la población y sirve como } \\
\text { puerta de entrada al sistema, que integra y coor- } \\
\text { dina la atención de salud, además de satisfacer } \\
\text { la mayor parte de las necesidades de salud de } \\
\text { la población. }\end{array}$ & - Telemedicina Intrahospitaria \\
\hline & $\begin{array}{l}\text { 4. Prestación de servicios especializados en el } \\
\text { lugar más apropiado, que se ofrecen de prefe- } \\
\text { rencia en entornos extra hospitalarios. }\end{array}$ & - Telemedicina Extrahospitalia \\
\hline & $\begin{array}{l}\text { 5. Existencia de mecanismos de coordinación } \\
\text { asistencial a lo largo de todo el continuo de los } \\
\text { servicios de salud. }\end{array}$ & $\begin{array}{l}\text { - Unidades lectoras de código } \\
\text { de barras, tecnologías de } \\
\text { identificación. } \\
\text { - Sistema de transporte sate- } \\
\text { lital }\end{array}$ \\
\hline & $\begin{array}{l}\text { 6. Atención de salud centrada en la persona, la } \\
\text { familia y la comunidad, a partir de las particu- } \\
\text { laridades culturales y de género, y los niveles de } \\
\text { diversidad de la población. }\end{array}$ & - Sistema CRM - Página Web. \\
\hline
\end{tabular}


Análisis del diseño de una red integrada de servicios de salud

\begin{tabular}{|c|c|c|}
\hline $\begin{array}{l}\text { Ámbitos de } \\
\text { abordaje }\end{array}$ & Atributos OPS & $\begin{array}{l}\text { Herramientas Logísticas/ } \\
\text { administrativas }\end{array}$ \\
\hline \multirow{3}{*}{ 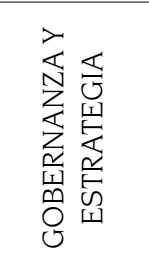 } & 7. Un sistema de dirección único para toda la red. & $\begin{array}{l}\text { - Cuadro de mando Integral } \\
\text { (CMI) }\end{array}$ \\
\hline & 8. Participación social amplia. & $\begin{array}{l}\text { - Sistema CRM - Redes socia- } \\
\text { les }\end{array}$ \\
\hline & $\begin{array}{l}\text { 9. Acción intersectorial y abordaje de los determi- } \\
\text { nantes de salud y la equidad en salud. }\end{array}$ & $\begin{array}{l}\text { - Transferencia electrónica de } \\
\text { datos (EDI) }\end{array}$ \\
\hline \multirow{4}{*}{ 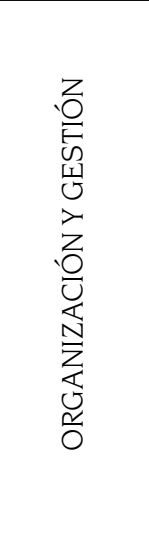 } & $\begin{array}{l}\text { 10. Gestión integrada de los sistemas de apoyo } \\
\text { administrativo, clínico y logístico. }\end{array}$ & $\begin{array}{l}\text { - Cuadro de mando Integral } \\
\text { (CMI) }\end{array}$ \\
\hline & $\begin{array}{l}\text { 11. Recursos humanos suficientes, competentes, } \\
\text { comprometidos y valorados por la red. }\end{array}$ & $\begin{array}{l}\text { - Sistema CRM - Planificación } \\
\text { del recurso humano necesa- } \\
\text { rio al diseño de la red y su } \\
\text { proyección futura. }\end{array}$ \\
\hline & $\begin{array}{l}\text { 12. Sistema de información integrado que vincula a } \\
\text { todos los miembros de la red, con desglose de } \\
\text { los datos por sexo, edad, lugar de residencia, } \\
\text { origen étnico y otras variables pertinentes. }\end{array}$ & $\begin{array}{l}\text { - Sistema CRM - Integrar el sis- } \\
\text { tema informático con todos } \\
\text { los miembros de la red. }\end{array}$ \\
\hline & 13. Gestión basada en resultados. & $\begin{array}{l}\text { - Cuadro de mando Integral } \\
\text { (CMI) }\end{array}$ \\
\hline 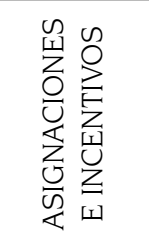 & $\begin{array}{l}\text { 14. Financiamiento adecuado e incentivos finan- } \\
\text { cieros alineados con las metas de la red. }\end{array}$ & $\begin{array}{l}\text { - Plataforma electrónica de } \\
\text { compras }\end{array}$ \\
\hline
\end{tabular}

Fuente: Pulgarín (2015, p. 89)

A continuación, se detallan las diferentes herramientas Logísticas/administrativas, para cada uno de los atributos:

\section{A. Implementación del modelo de gestión CRM}

A partir del análisis e inferencia del proceso de la cadena de suministro, la estrategia CRM debe incorporarse en los atributos 1, 2, 6, 8, 11 y 12 del modelo de RISS establecido por la OPS, dado que modifican de manera directa la logística de los subsistemas de la red en cuanto a las relaciones que se establecen con cada uno de los actores (ver figura 4).

El modelo de gestión de la organización, enfocado al cliente es conocido en el mercado como customer relationship management (CRM), el cual, se fundamenta en la gestión basada en adecuar la oferta a los gustos de los clientes y satisfacer sus 
necesidades; para alcanzar este fin se crea una base de datos que contenga perfiles completos. Por otra parte, el sistema de gestión CRM permite obtener mayor información sobre los usuarios lo que genera relaciones a largo plazo, atrae nuevos pacientes, mejora la comunicación y aumenta el grado de satisfacción; este sistema de gestión también hace más sencillo el proceso de remisión de pacientes entre médicos, especialistas, departamentos o estudios especiales, pues al facilitar la comunicación se puede ayudar a los pacientes a recibir atención continua y disminuye la carga de trabajo administrativo.

Atributo $\mathbf{N} .^{\circ} \mathbf{1}$ : el sistema de gestión permite contar con una base de datos sobre la población actualizada y de esta manera es posible tener conocimiento de los servicios más demandados dentro de la red y los medicamentos requeridos para el tratamiento; al contar con esta información se realiza una planificación actual y futura de los servicios de salud y de atención a las personas. Respecto a la dinámica de la cadena de suministro la propuesta es permitir el acceso de los proveedores a la base de datos a través del cloud computing y de esta manera lograr que sean los proveedores los que se encarguen de mantener los niveles de inventarios permitidos y garantizar el suministro continuo de bienes hospitalarios a los diferentes puntos de la red.

Atributo $\mathbf{N}^{\circ}{ }^{\circ} \mathbf{2}$ : en este atributo es indispensable adaptarse a los requerimientos locales; el sistema CRM para salud permite establecer segmentos específicos para diferentes programas. Los pacientes son agrupados a partir de las causas como la demografía, psicográficos y de alto riesgo, problemas de salud y otros lo que permite mayor flexibilidad de la cadena de suministro y orientar con precisión la prestación de servicio a grupos de pacientes con requerimientos determinados o procedimientos específicos.

Atributo $\mathbf{N}^{\circ}$ 6: el sistema de gestión CRM permite alcanzar un enfoque familiar y comunitario en la dispensación de servicios y tratamientos, participación de la comunidad en la identificación de las necesidades de salud, la planeación y la entrega de los servicios. En este punto la herramienta a utilizar es la implementación de la página web de la red de servicios de salud, la cual permite programación de citas y la incorporación de la comunidad en el mejoramiento de los servicios de salud.

Atributo N. ${ }^{\circ}$ 8: la participación social amplia está vinculada con el sistema de gestión CRM, y para este atributo se deben utilizar como herramientas las diferentes redes sociales con las cuales interactúa la comunidad; de esta manera se podrá estimular la capacidad de autocuidado de los usuarios, y entender y considerar las inquietudes y aspiraciones del público para lograr un empoderamiento de los mismos con la red prestadora de servicios de salud. 
Atributo N. ${ }^{\circ}$ 11: la base de datos creada en el cloud computing (almacén de datos) con el sistema de gestión CRM permite determinar tendencias marcadas en la demanda de los usuarios en servicios de salud por lo cual el recurso humano será suficiente, competente y comprometido con red.

Atributo N. ${ }^{\circ}$ 12: el sistema de gestión de la información es necesario dentro del sistema de gestión CRM y permite, a través del cloud computing, que todos los miembros de la red tengan acceso a la misma desde cualquier ubicación geográfica para obtener los datos relevantes dentro de su perfil de desempeño.

Figura 4. Mecanismo de integración basado en la tecnología CRM

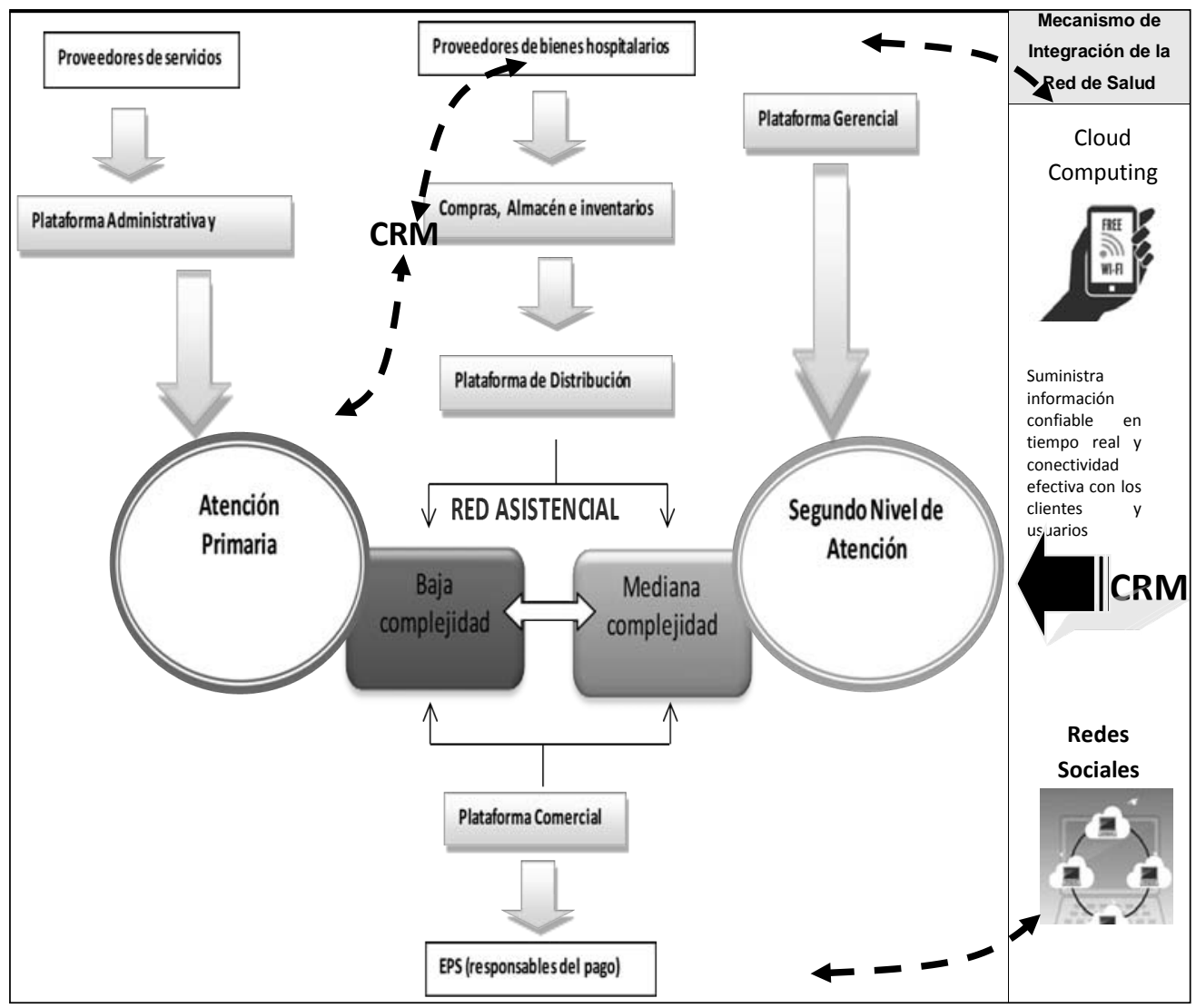

Fuente: Elaboración propia a partir de Pulgarín (2015, p. 94)

\section{B. La telemedicina}

La telemedicina es una herramienta de la logística hospitalaria que permite que en el primer nivel de atención el paciente tenga acceso a especialistas para brindar una 
atención multidisciplinaria y evitar el traslado del paciente al punto principal de la red (ver figura 5). La herramienta comunica audiovisualmente y permite la transferencia de imágenes entre distintos puntos de la red de salud a través de aplicaciones como Skype, MeetingBurner y Facebook.

Atributo N. ${ }^{\circ}$ 3: se plantea un modelo de atención de telemedicina intrahospitalaria donde el centro de atención de especialistas debe estar ubicado en el alma máter de la red, y las patologías a tratar por este medio dependen de la caracterización de la población y la capacidad de la red; por ejemplo, en la ESE Oriente debe de estar ubicado en el Hospital Carlos Holmes Trujillo.

Atributo $\mathbf{N}^{\circ}$ 4: la telemedicina también permite tratar problemas de salud en entornos extrahospitalarios y de esta manera lograr que llegue la atención de especialistas a lugares de difícil acceso ya sea por su situación social o geográfica.

Figura 5. Telemedicina extra e intrahospitalaria

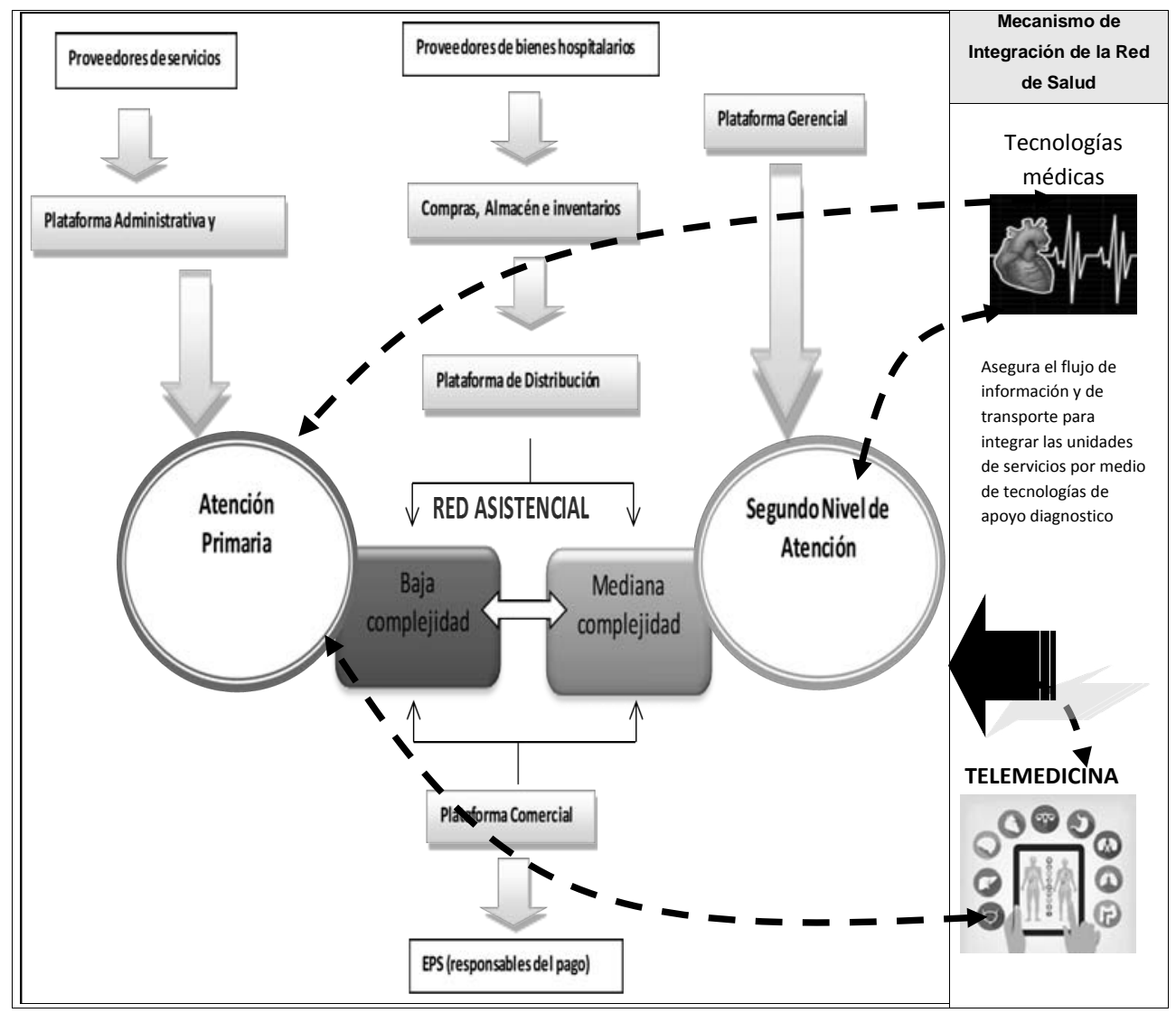

Fuente: Elaboración propia a partir de Pulgarín $(2015$, p. 95) 


\section{Mecanismos de coordinación asistencial}

Una herramienta que permite el control y coordinación del transporte es el sistema de rastreo satelital, ya que se puede tener conocimiento del tiempo y del lugar en el que se encuentra la flotilla de ambulancias, evitar rutas con bloqueos o retrasos que impacten en la asistencia oportuna al paciente, calcular el desgaste de partes del vehículo para efectuar mantenimiento preventivo, entre otros. Este tipo de datos también facilita controlar al personal y ayuda a reducir costos.

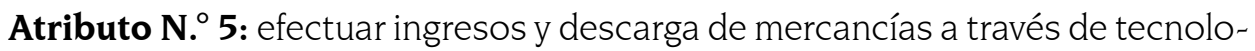
gías de identificación permite que todos los integrantes de la red mantengan al día los inventarios, y de esta manera no incurran en faltantes o excesos de productos. Es importante en este punto que la información fluya entre los proveedores y la red por medio de un almacén de datos (cloud computing) que permitirá monitorizar los inventarios conforme lo establecido en el atributo 1. Entre los muchos beneficios de estas tecnologías vale mencionar su impacto en el servicio que disminuye los tiempos de recepción física y administrativa de los medicamentos; por lo tanto, el personal logrará dedicar más tiempo a sus procesos misionales, y la red integrada de servicios de salud podrá mejorar los tiempos y disminuir errores en la facturación (ver figura 6).

\section{Figura 6. Mecanismos de coordinación asistencial}

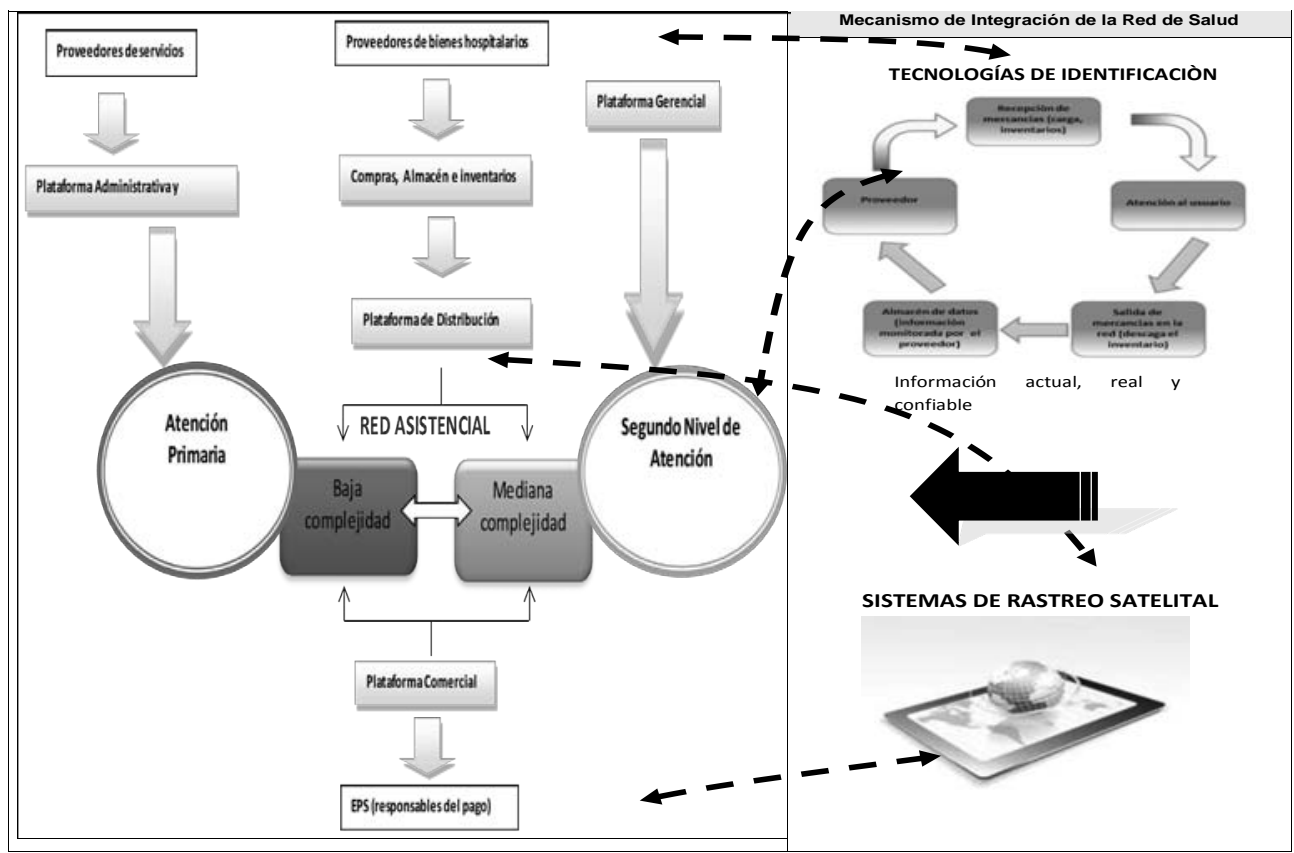

Fuente: Ēlaboración propia a partir de Pülgarín $(2015, \bar{p} .98)$ 


\section{Cuadro de mando integral}

La herramienta que responde a los requerimientos de los atributos 7, 10 y 13 de la RISS (ver figura 7) es el cuadro de mando integral (CMI). Esta es una herramienta de gestión administrativa que permite llevar a cabo la monitorización y evaluación de la red con el fin de prestar un nivel de servicio apropiado. A través del CMI se logra alinear las personas y los recursos bajo la misma misión, visión, valores y principios compartidos por las partes de la red; para que el CMI funcione se deben establecer las perspectivas y los indicadores de la misma:

* Perspectiva de aprendizaje: este concepto es vital en un sistema de salud, puesto que tiene que ver directamente con los recursos que más contribuyen a la generación de valor: las personas y la tecnología; por lo tanto, esta perspectiva se ocupa de realizar constantes capacitaciones al personal, al igual que implementar tecnologías que permitan una gestión integrada de las diferentes partes que conforman la red. Dentro de los indicadores a tener en cuenta bajo este concepto: 1. Antigüedad del personal. 2. Gastos de capacitación. 3. Inversión en TIC. 4. Ausentismo. 5. Encuestas de clima organizacional.

* Perspectiva del proceso interno: este punto está relacionado con las mejoras o innovaciones en los procesos internos de la red; por lo tanto, se busca identificar los procesos críticos que impactan en la satisfacción del cliente. Los indicadores de este punto van enfocados al tiempo, calidad y costo de las operaciones internas de la red: 1. Demora en el servicio al cliente. 2. Satisfacción del cliente. 3. Rotación de inventarios. 4. Porcentaje errores de facturación. 5. Roturas de stock.

* Perspectiva del cliente: se refiere al conocimiento y segmento de la comunidad a la cual se prestan los servicios. Dentro de los indicadores que se deben tener en cuenta: 1. Captación de pacientes. 2. Cuota de mercado. 3. Tasa de crecimiento de pacientes.

* Perspectiva financiera: en esta última perspectiva se ven reflejados los resultados de las estrategias y objetivos fijados por la red en las tres perspectivas anteriores. El objetivo que busca este último punto es la creación de valor para los clientes, empleados y la red, además del equilibrio aceptable. Algunos de los indicadores a tener en cuenta: 1. Rentabilidad económica. 2. Nivel de apalancamiento. 3. Razón corriente. 4. Rentabilidad del patrimonio. 5. Capital neto de trabajo. 
Figura 7. Cuadro de mando integral

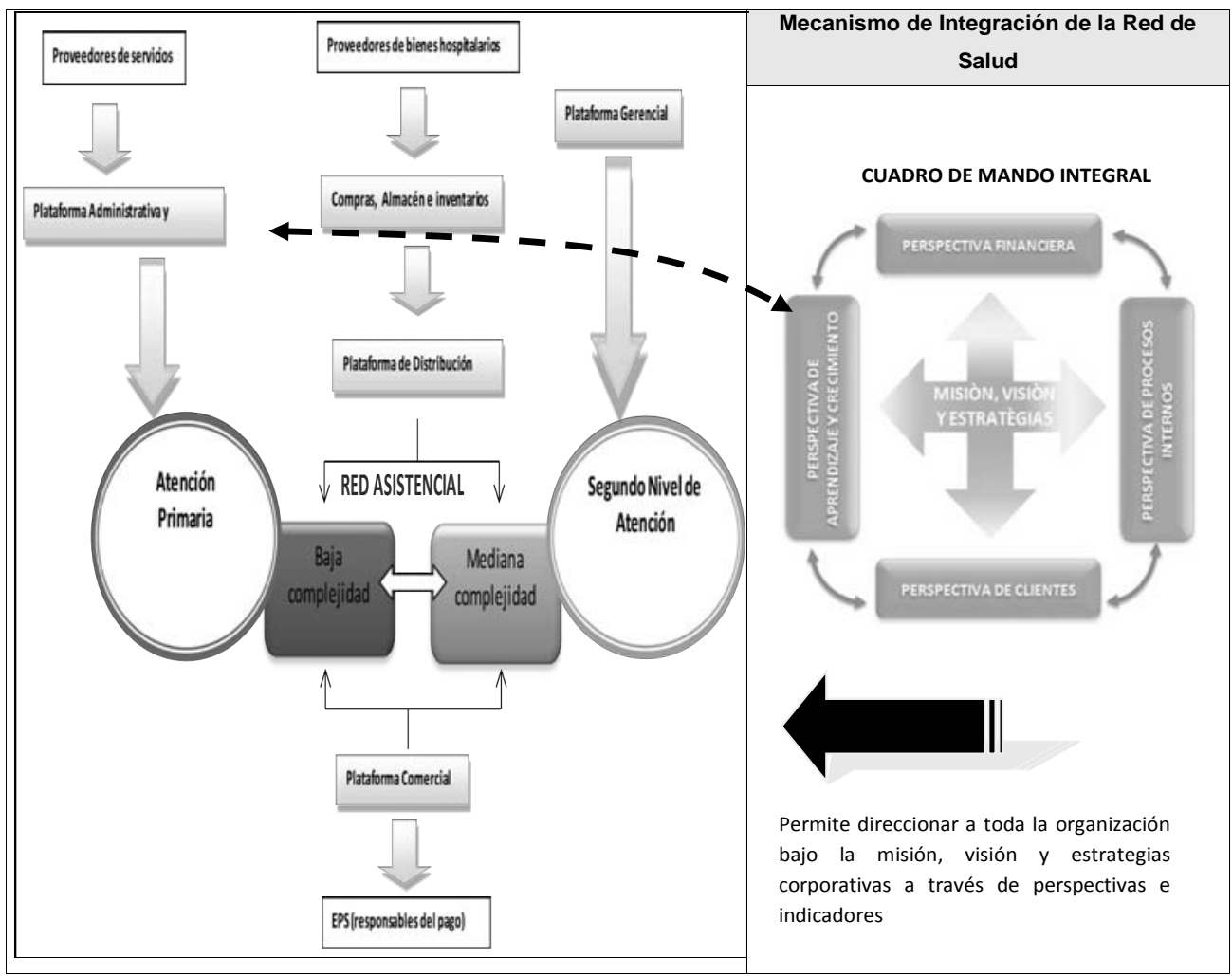

Fuente: Elaboración propia a partir de Pulgarín (2015, p. 99)

\section{E. Transferencia electrónica de datos (EDI)}

Las redes integradas de servicios de salud (RISS), por su naturaleza, están relacionadas con múltiples actores de la comunidad y el Estado, por ejemplo, bancos, contralorías, proveedores, ICBF, medicina legal, INVIMA, Ministerio de Salud, instituciones educadoras, entre otras. 
Figura 8. Transferencia electrónica de datos (EDI)

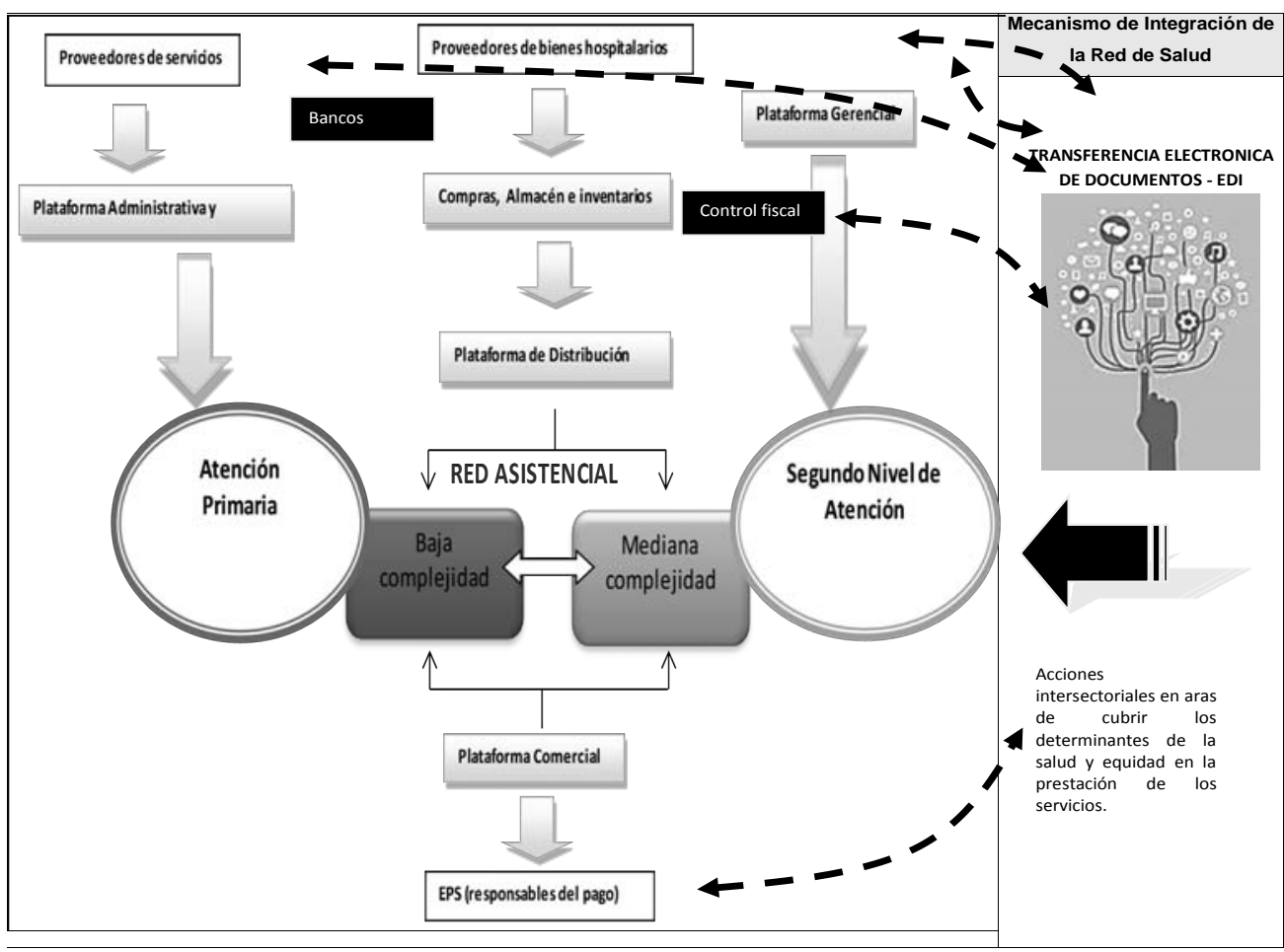

Fuente: Elaboración propia a partir de Pulgarín (2015, p. 101)

Atributo N. ${ }^{\circ}$ 9: lo anterior implica la acción intersectorial, e incluye una colaboración con diferentes instituciones y organizaciones del sector público y privado; en este sentido un mecanismo de integración de la red se obtiene a través de la transferencia electrónica de datos; sin embargo, la transferencia electrónica de datos se debe establecer entre los actores más representativos de la red, como las EPS, los proveedores, la contraloría, entidades bancarias. También se deben tener en cuenta los beneficios de la transferencia electrónica de datos ya que permite agilizar los procesos, disminuye las transacciones sobre papel y la intervención del personal; por lo tanto, se reduce la probabilidad de error en los documentos y los costos administrativos (ver figura 8).

\section{F. Plataforma electrónica de compras}

La plataforma electrónica de compras para la adquisición de insumos en las instituciones hospitalarias se constituye en un mecanismo efectivo de integración de red, debido a la gestión de compras centralizadas entre varias instituciones prestadoras 
de salud sean o no parte de la red en cuestión, y se encuentra reglamentada por la Ley 1438 de 2011, artículo 76 (CRC, 2011).

Atributo $\mathbf{N} .^{\circ}$ 14: estas plataformas de compras permiten que los vendedores obtengan información sobre las necesidades reales de los compradores y de esta manera se evitan situaciones como el efecto látigo que perjudica la eficiencia de la cadena de suministro, el cual, Mejía, Palacio y Adarme (2013, p. 41), lo definen como "una distorsión creciente de la demanda transmitida por los distintos agentes participantes en la gestión del flujo de productos a medida que nos alejamos del mercado". De igual manera tanto el comprador como el vendedor pueden efectuar una trazabilidad de los pedidos en tiempo real; por lo cual logran monitorizar el estado, entrega y pago de la mercancía. Para poder implementar esta herramienta es necesario que todos los implicados en la cadena de suministro estén comprometidos y cuenten con un mismo lenguaje de comunicación informática (ver figuras 9 y 10).

Figura 9. Plataforma electrónica de compras

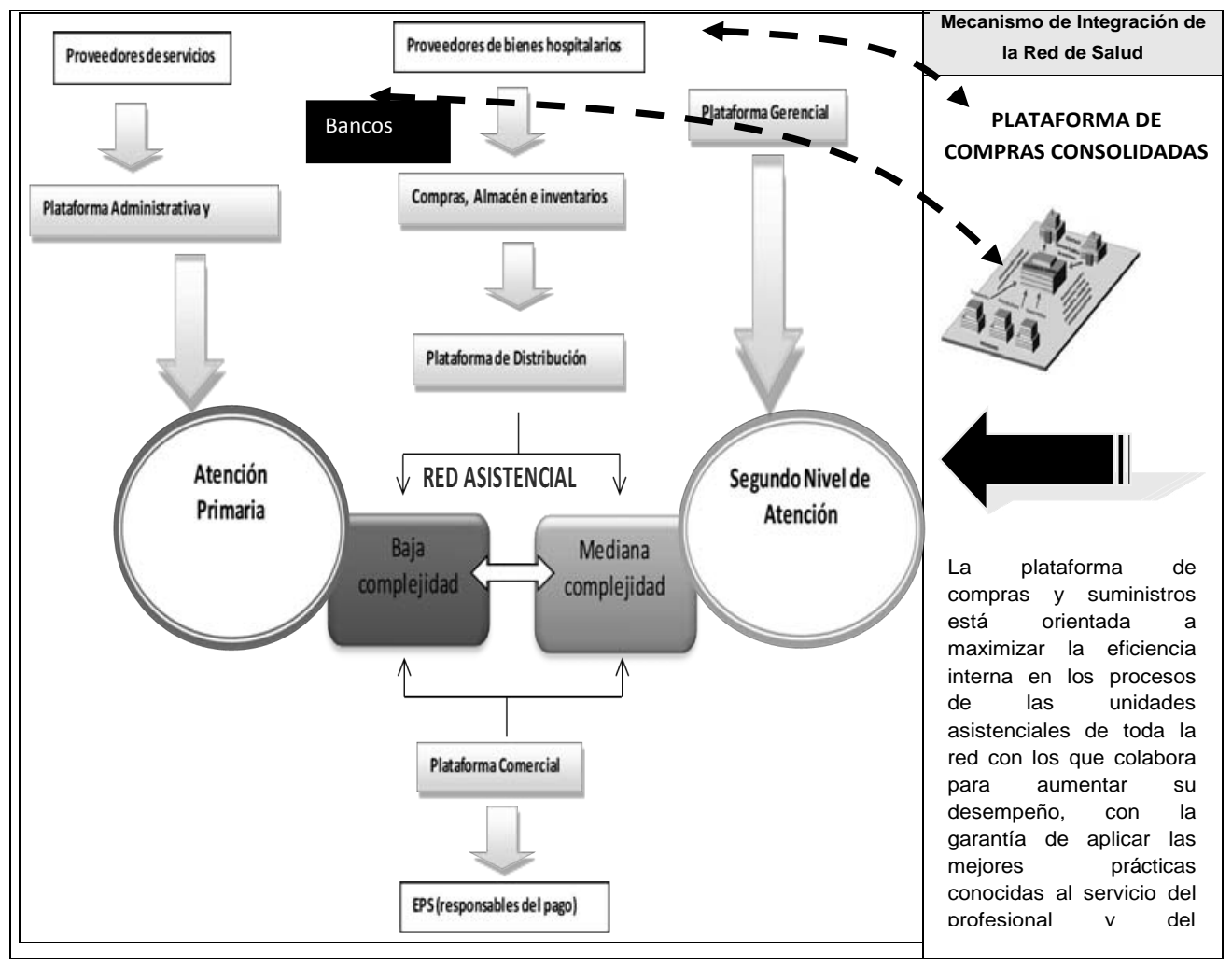

Fuente: Elaboración propia a partir de Pulgarín (2015, p. 102) 
Figura 10. Plataforma de compras y suministros para la Red

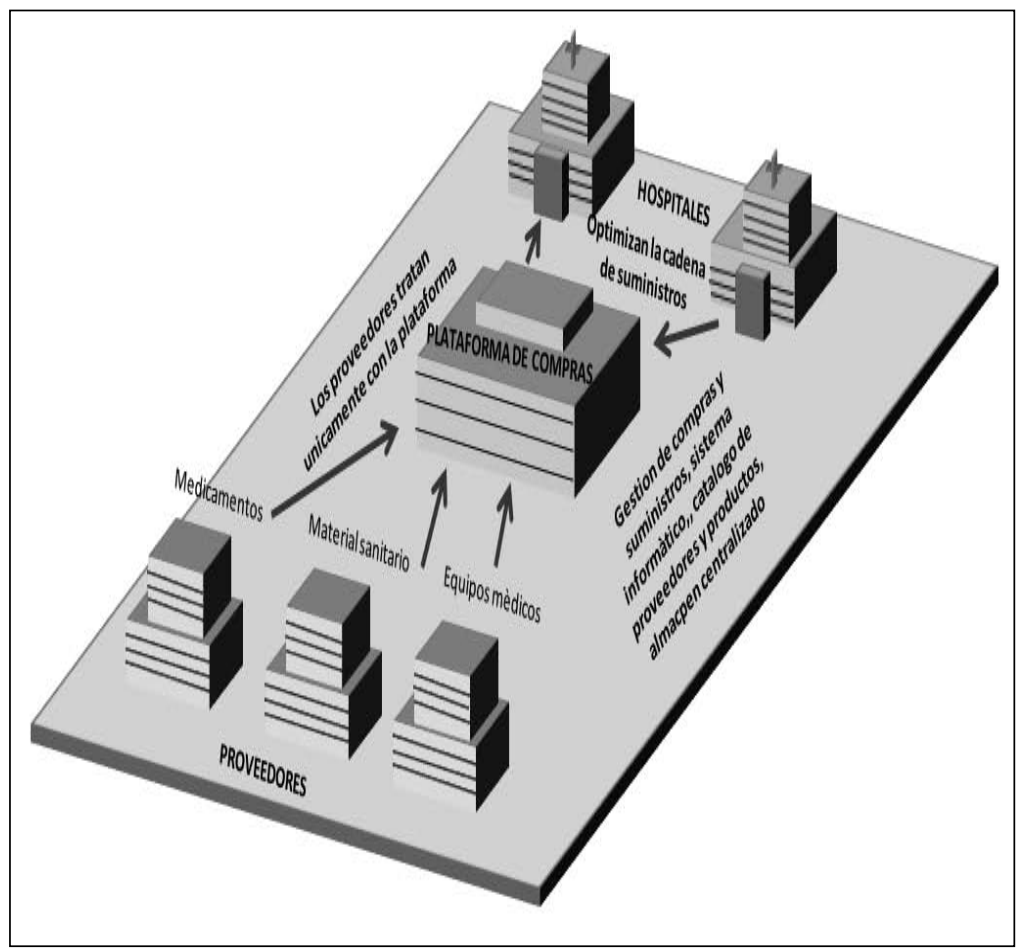

Fuente: Pulgarín $(2015$, p. 103)

\subsection{Aplicación del análisis de redes}

El análisis de redes es un instrumento metodológico que permite simular, de manera directa, la dinámica de una red, cuantificar los actores en forma matricial y visualizar la estructura en red. La esencia del instrumento metodológico está constituida por el álgebra matricial y los grafos; para tal fin se utiliza un programa básico para el análisis de redes llamado Ucinet 6.0.

Con el fin de obtener un análisis riguroso se aplican los pasos ya validados por Tabarquino (2015, p. 228-230): i) Se determina el número de actores involucrados en la red y se categoriza su naturaleza y peso a partir de Marsh y Rhodes (1992); Jordana (1995); Börzel (1997); Klijn (1998); Porras (2001); Petrizzo (2004); Musso, (2004); Zurbriggen (2011); ii) se construye las matrices de actores (la existencia de vínculo es el valor de uno y si no existe un vínculo el valor será cero) y posteriormente se insertan en el programa de Ucinet 6.0, con el fin de visualizar la dinámica en Red; y iii) para el análisis de la estructura en Red se utiliza el programa de redes Ucinet 6.0 para calcular la densidad, grado nodal -degree-, indicadores descriptivos -media, desviación estándar e índice de centralización-, grado de intermediación -between- 
ness-, grado de la cercanía -closenness-, si la matriz es simétrica, y si no es simétrica se calcula el Power Bonicint, a partir de Lozares (1996, 2005); Molina (2004); Molina y Ávila (2009); Quiroga (2003); Sanz (2003); Velázquez y Aguilar (2005); Wasserman y Faust, (1994); Borgatti (2003); Garrido (2001).

\subsubsection{Identificación y caracterización de actores involucrados en la red}

Los actores involucrados en la red de la cadena de abastecimiento de la ESE Oriente de Cali se clasifican en hospital, centro de salud, puesto de salud, proveedor minorista y proveedor mayorista (ver tabla 5).

Tabla 5. Actores involucrados en la red de la cadena de suministro de la ESE Oriente de Cali

\begin{tabular}{|c|c|c|}
\hline $\mathrm{N}^{o}$ & Nombre y atributo & Área de influencia dentro de la red \\
\hline 1 & Hospital Carlos Holmes Trujillo & Comunas $13,14,15$ y 21 \\
\hline 2 & Centro de Salud El Diamante & \multirow{9}{*}{ Comuna 13} \\
\hline 3 & Puesto de Salud Calipso & \\
\hline 4 & Puesto de Salud Comuneros II & \\
\hline 5 & Puesto de Salud Los Lagos & \\
\hline 6 & Puesto de Salud Ricardo Balcázar & \\
\hline 7 & Puesto de Salud Charco Azul & \\
\hline 8 & Puesto de Salud Ulpiano Lloreda & \\
\hline 9 & Puesto de Salud El Poblado II & \\
\hline 10 & Puesto de Salud El Vergel & \\
\hline 11 & Centro de Salud Marroquín Cauquita & \multirow{6}{*}{ Comuna 14} \\
\hline 12 & Centro de Salud Manuela Beltrán & \\
\hline 13 & Puesto de Salud Alirio Mora Beltrán & \\
\hline 14 & Puesto de Salud Alfonso Bonilla Aragón & \\
\hline 15 & Puesto de Salud Los Naranjos & \\
\hline 16 & Puesto de Salud Intervenidas & \\
\hline 17 & Centro de Salud El Vallado & \multirow{5}{*}{ Comuna 15} \\
\hline 18 & Puesto de Salud Comuneros & \\
\hline 19 & Puesto de Salud Ciudad Córdoba & \\
\hline 20 & Puesto de Salud Mojica & \\
\hline 21 & Puesto de Salud El Retiro & \\
\hline
\end{tabular}


Dora Marcela Pulgarín Henao - Raúl Andrés Tabarquino Muñoz

\begin{tabular}{|c|c|c|}
\hline $\mathrm{N}^{o}$ & Nombre $y$ atributo & Área de influencia dentro de la red \\
\hline 22 & Centro de Salud Decepaz & \multirow{4}{*}{ Comuna 21} \\
\hline 23 & Centro de Salud Potrero Grande & \\
\hline 24 & Puesto de Salud Pizamos & \\
\hline 25 & Puesto de Salud de Navarro & \\
\hline 26 & Proveedor Mayorista Adolfo Allers & \multirow{8}{*}{ Comunas $13,14,15$ y 21} \\
\hline 27 & Proveedor Mayorista Droguería Alianza & \\
\hline 28 & Proveedor Mayorista Equipadora Médica & \\
\hline 29 & Proveedor Mayorista Roche & \\
\hline 30 & Proveedor Mayorista LaFrancol & \\
\hline 31 & Proveedor Minorista Aseo Buriticá & \\
\hline 32 & Proveedor Minorista Institucional & \\
\hline 33 & Proveedor Minorista Papelería Andina & \\
\hline
\end{tabular}

Fuente: Elaboración propia a partir de Pulgarín (2015, p. 56-61)

\subsubsection{Visualización de la estructura en red}

Después de identificar y caracterizar de los 33 actores involucrados en la red de la cadena de suministro de la ESE Oriente de Cali, se organizan en forma matricial de $33 \times 33$, en donde uno (1) representa la existencia de vínculo, y cero (0) la no existencia de vínculo; el ejercicio de matricial se realizó partiendo de los resultados obtenidos por Pulgarín (2015), por lo cual, se obtuvieron tres matrices: i) sin la aplicación del concepto de RISS; ii) con la aplicación del concepto de RISS y iii) con los 14 atributos de la RISS. Posteriormente las matrices obtenidas se introducen en el programa de análisis de redes Ucinet, con el fin de visualizar la dinámica en Red (ver anexos salidas de matrices de Ucinet 6.0)

\section{A. Sin la aplicación del concepto de la RISS}

Al observar el comportamiento de la red a partir de la cadena de suministro sin la aplicación del concepto de RISS, se evidencia que el actor principal es el Hospital Carlos Holmes Trujillo, por ser el encargado de realizar los pedidos de toda la red tanto a los cinco mayoristas como a los tres minoristas, y a su vez, los distribuye a los centros de salud y estos últimos se encargan de coordinar la distribución de los suministros a los puestos de salud de su área de influencia. 
Gráfico 1. La red de la cadena de suministro de la ESE Oriente de Cali sin la aplicación del concepto de RISS

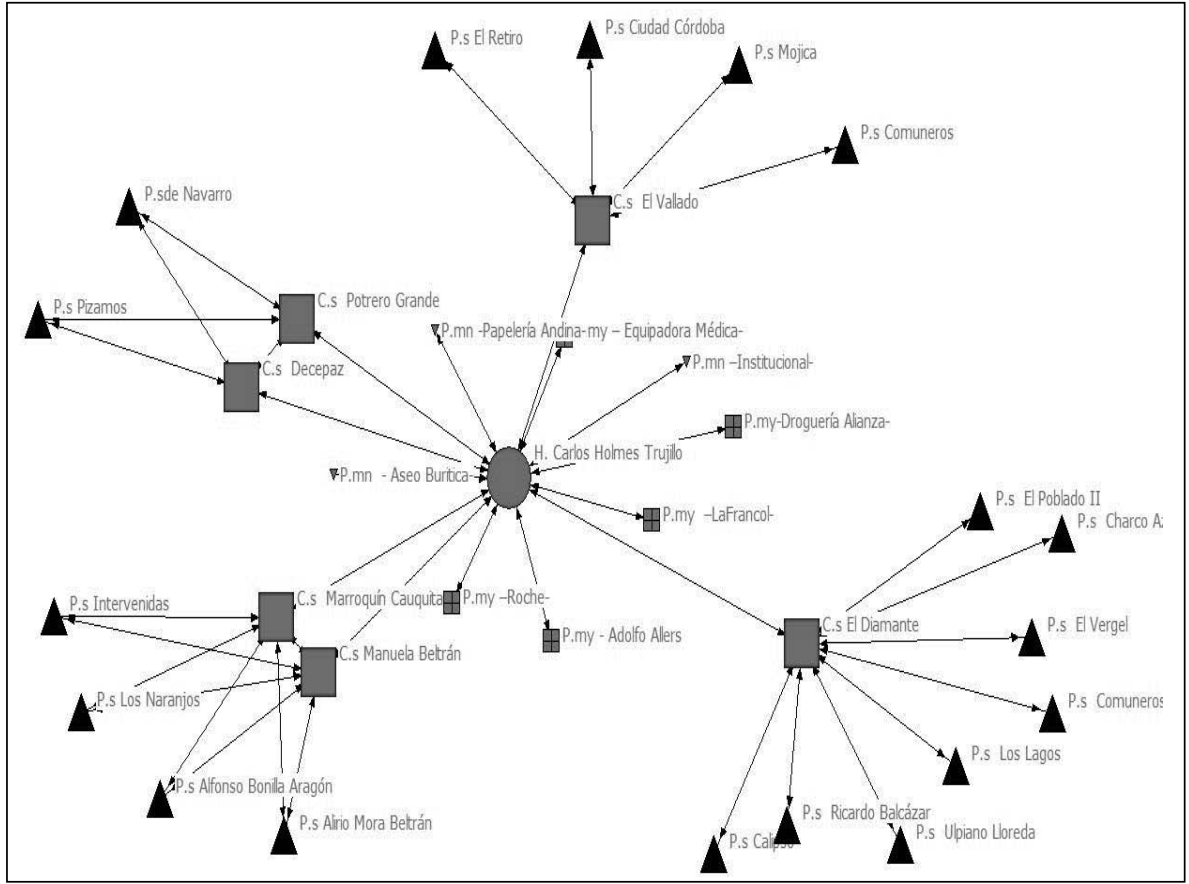

Fuente: Elaboración propia con Ucinet 6.0 a partir de los datos de la tabla 6

\section{B. Con la aplicación del concepto de la RISS}

Al observar el comportamiento de la red a partir de la cadena de suministro aplicando el concepto de la RISS, se observa que: i) los puestos de salud dentro de la nueva estructura de la red ganan importancia, en el sentido que ya no dependen de los suministros de parte del hospital Carlos Holmes Trujillo, sino que esta tarea que no hace parte del Cor del hospital como tal, la van llevar a cabo a través de una plataforma con los diferentes proveedores; ii) Los proveedores van a tener una relación directa, además del hospital, con puestos de salud y centros de salud a través de una plataforma en donde se encargarán de suministrar y abastecer todo tipo de suministros a través de políticas de almacenamiento que debe de emitir el hospital, a partir de la demanda y las proyecciones que tiene para los diferentes periodos $\mathrm{O}$ diferentes años; y iii) la relación puestos de salud y centro de salud en relación a suministros ya no va ser directa; se mejora así la prestación del servicio en ambos. 
Grafico 2. La red de la cadena de suministro de la ESE Oriente Cali aplicando el concepto de RISS

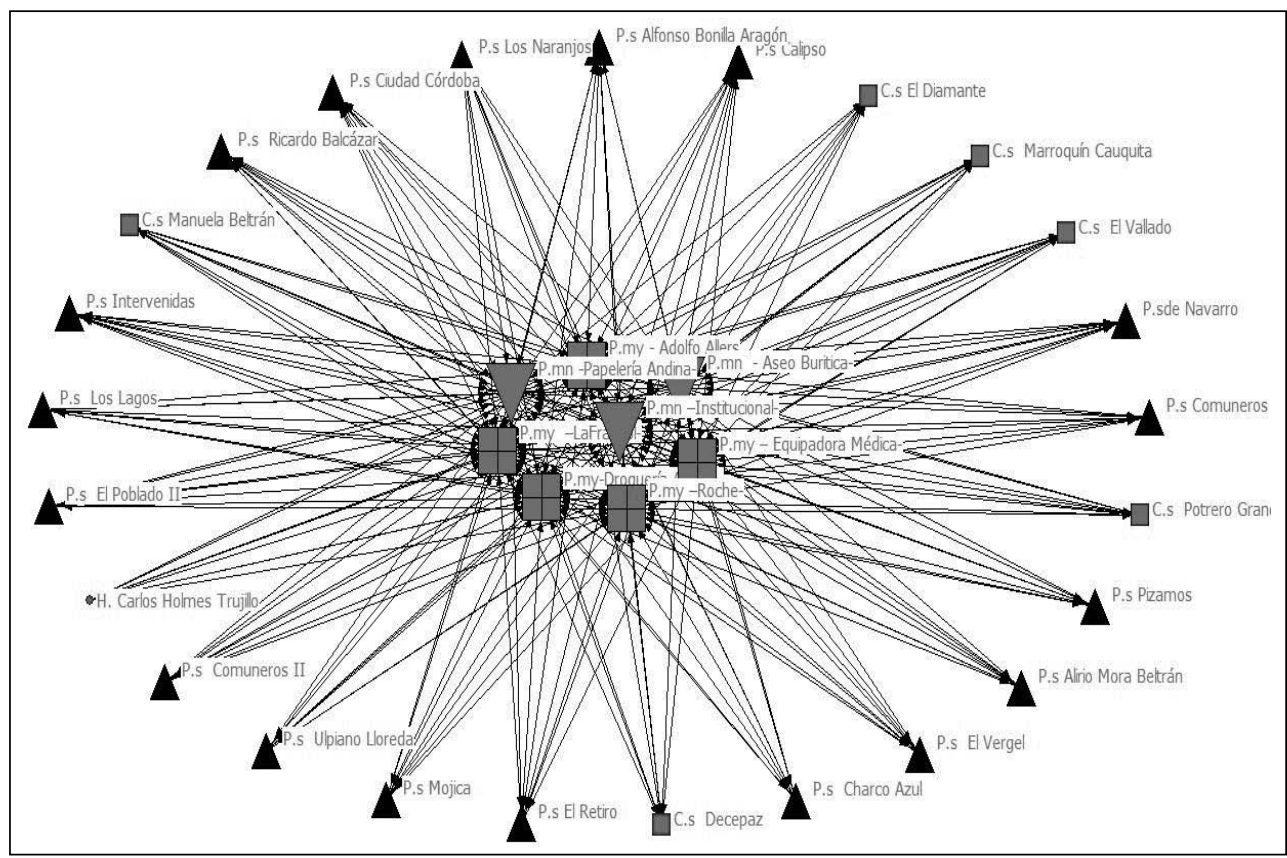

Fuente: Elaboración propia con Ucinet 6.0 a partir de los datos de la tabla 7

\section{Aplicación de los 14 atributos mínimos de la RISS}

Al observar el comportamiento de la red a partir de la aplicación de los 14 atributos mínimos de la RISS según la OPS (2010), se observa que: i) los puestos de salud que solo se conectaban con los centros de salud en la anterior estructura de red, se conectan ahora con el Hospital Carlos Holmes Trujillo, debido a que la nueva herramienta de conectividad es la telemedicina ya que los especialistas que se encuentran en el Hospital Carlos Holmes Trujillo van a poder asesorar a los puestos de salud para que brinden servicios especializados; ii) La función de la distribución se dinamiza en varios nodos, mejora la prestación del servicio en relación con que los usuarios podrán desplazarse en varios puntos aprovechando la infraestructura de los centros de salud; y iii) La plataforma va estar en la nube, por ende, esta se encuentra dentro de toda la red, y los diferentes actores tendrán acceso a ella. 
Gráfico 3. La red de la de la ESE Oriente Cali aplicando los 14 atributos mínimos de la RISS

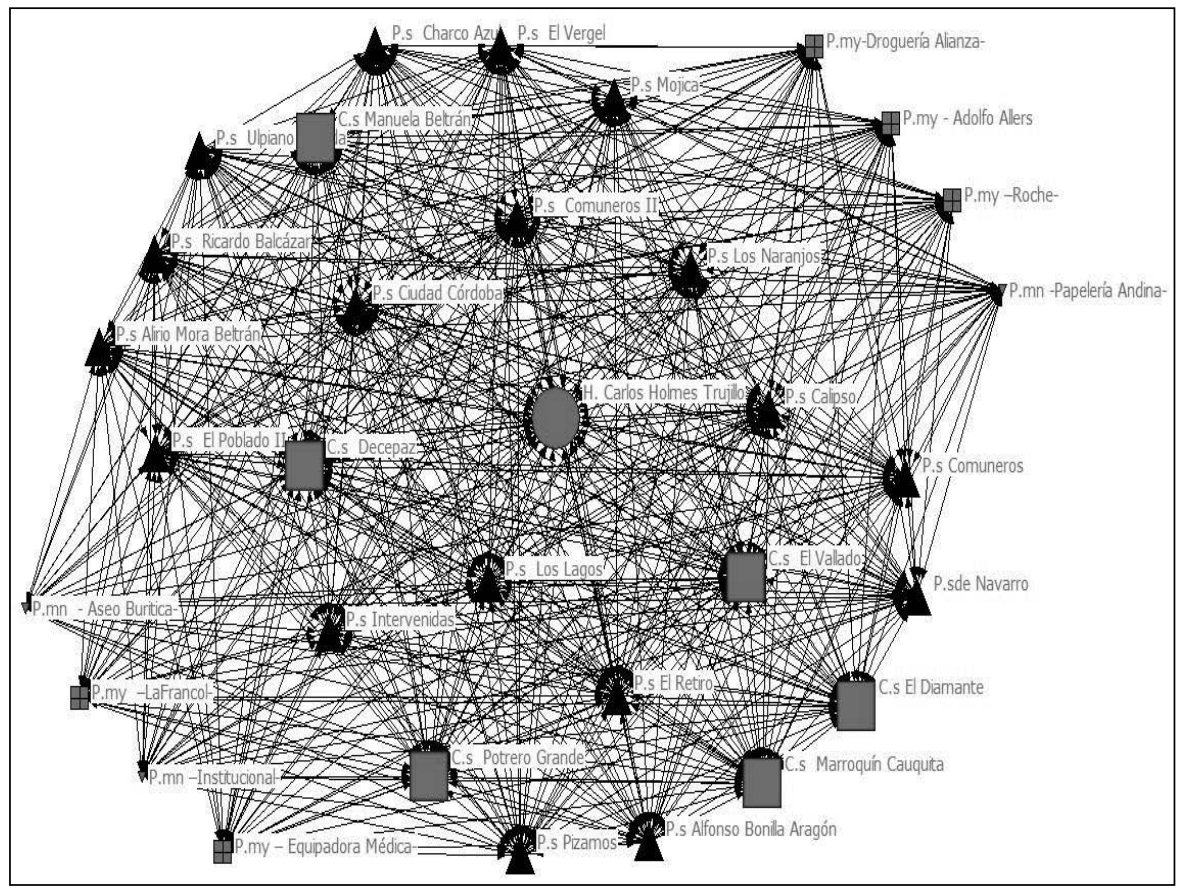

Fuente: Elaboración propia con Ucinet 6.0 a partir de los datos de la tabla 8.

\subsubsection{Análisis de la estructura en Red}

Para realizar el debido analisis de la estructura en red, se calculan indicadores de relaciones posibles ${ }^{1}$, densidad ${ }^{2}$, rango, media, desvicion estándar, índice de centralizacion, el grado de intermediacion y de cercanía, según Tabarquino (2015).

Tabla 9. Análisis de la estructura en Red de la ESE Oriente antes y después de la RISS

\begin{tabular}{|l|l|l|l|}
\hline \multirow{2}{*}{} & \multicolumn{2}{|c|}{$\begin{array}{c}\text { Red de Cadena de Suministro de la ESE Oriente de } \\
\text { Cali }\end{array}$} & $\begin{array}{c}\text { Red de la ESE Oriente } \\
\text { Aplicando los 14 atributos }\end{array}$ \\
\cline { 2 - 3 } & Antes de la RISS & Después de la RISS & \\
\hline $\begin{array}{l}\text { El total de las re }- \\
\text { laciones posibles } \\
\text { de la red }\end{array}$ & 1056 & \multicolumn{2}{|}{} \\
\hline
\end{tabular}

- Se calcula multiplicando el número total de nodos por el número total de nodos menos uno $33 \times(33-1)=1056$.

2 Se calcula de manera manual dividiendo el número de relaciones existentes entre las posibles y multiplicado por 100 (A/1056 x100 = B \%). 


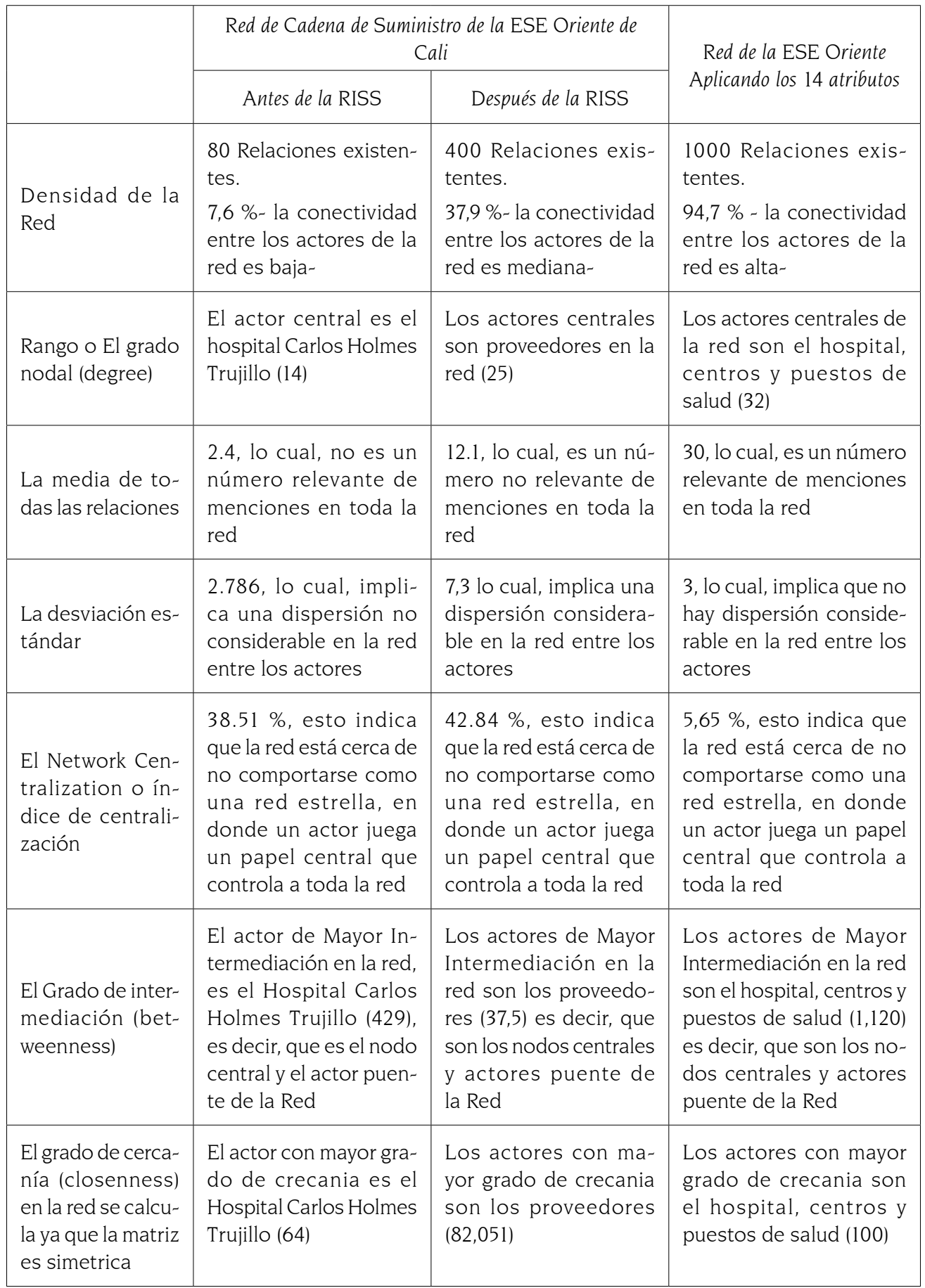

Fuente: Elaboración propia 


\section{CONCLUSIONES}

Las redes integradas de servicios de salud se implementan en Colombia a través del MSPSC (2016, p. 4) con la Resolución 429 bajo la denominación de redes integrales de prestadores de servicios de salud (RED).

La medición de los atributos de la RISS debe contar con series históricas consolidadas, que determinen la población objeto de la red y permitan realizar el contraste con el portafolio de servicios de la red.

Para una evaluación adecuada de los atributos de una red integrada de servicios de salud, se debe proyectar con datos reales y de base de la pirámide poblacional del territorio atendida por la organización o institución que es objeto de estudio; de lo contrario se partirá de bases especulativas sobre la capacidad de reacción de la red.

Para evaluaciones posteriores de redes integradas de servicios de salud, es pertinente la complementación de la herramienta del SCOR model con la del análisis de redes, ya que esta última permite visualizar y analizar cómo funciona la dinámica de una red organizacional y, a su vez, el modelo SCOR facilita la definición de los insumos principales de la red.

El modelo de gestión y desarrollo de una RISS debe apuntar a los planteamientos de la gestión de redes, que no solo implica una buena cadena de suministro en la adquisición de materiales (compras), mejoras en las condiciones financieras, de entrega y calidad, sino también en el diseño de estrategias de la organización que impacta en los costos operativos de la red.

La amplitud de los recursos destinados a las operaciones logísticas en el sector salud genera dificultades a los profesionales para implantar modelos que permitan mejorar su desempeño; estos obstáculos se deben, en gran parte, a que existen demasiados departamentos que manejan temas logísticos (abastecimiento, droguería, servicios auxiliares, internación, etc.), por lo cual, el área logística se debe integrar con el área estratégica de los hospitales.

\section{BIBLIOGRAFÍA}

Aguilar, Víctor; Garrido, Pedro y Gallego, Godino (2013). Mejorando la cadena de suministro en un hospital mediante la gestión Lean. En: Revista de Calidad Asistencial, Vol. 28, No. 6, p. 337-344.

Aguilar, Víctor y Garrido, P. (2013). Gestión Lean en logística de hospitales: estudio de un caso. En: Revista de Calidad Asistencial, Vol. 28, No. 1, p. 42-49.

Agudelo, Carlos; Cardona, Jaime; Ortega, Jesús y Robledo, Rocío (2011). Sistema de salud en Colombia: 20 años de logros y problemas. En: Revista Ciência E Saúde Coletiva, Vol. 16, No. 6, p. 2817-2828. 
Amaya, Ciro; Beaulieu, Martín; Landry, Sylvain; Rebolledo, Claudia y Velasco, Nubia (2010). Potenciando la contribución de la logística hospitalaria: tres casos, tres trayectorias. En: Revista Gestión Internacional, Vol. 14, No. 4, p. 85-98.

Artaza, Oswaldo; Méndez, Claudio; Holder, Reynaldo y Suárez, Julio Manuel (2011). Redes integradas de servicios de salud: el desafío de los hospitales. Documentos OPS/OMS en Chile No. 1. Santiago, Chile: OPS/OMS, 304p.

Arévalo Tamayo, Javier (2014). Gerente Red de Salud Oriente-Empresa Social del Estado-. Santiago de Cali, Entrevista, julio 1 del 2014.

Barragán Bechara, Juan Carlos; Riaño Casallas, Martha Isabel y Martínez, Magda (2012). Redes integradas de servicios de salud: hacia la construcción de un concepto. En: Revista Universidad y Salud. Vol.14, No. 2, p. 186-196.

Balladelli, Pier Paolo (2013). Redes Integradas de Servicios de Salud y Atención en Salud Mental. Ponencia presentada en la Asamblea Provincial del Capítulo 2010-2014 de la Orden Hospitalaria de San Juan de Dios. Buenos Aires, Argentina, 21p.

Beery, Moira y Vallianatos, Mark (2004). Farm to hospital: Promoting health and supporting local agriculture. UEPI Papers, 13p.

Borgatti, Steve (2003). Conceptos básicos de redes sociales. Analytic Technologies. Cancún, México, 4lp.

Börzel, Tanja (1997). Qué tienen de especial los policy networks? Explorando el concepto y su utilidad para el estudio de la gobernanza europea. Instituto Universitario Europeo en Florencia, 50p.

Breen, Liz (2004) Is there a place for Third Party Logistics providers in the NHS pharmaceutical supply chain in Hospital Pharmacy? The case of Central Manchester and Manchester Children's University Hospitals NHS Trust. En: Hospital Pharmacist, No. 11, p. 33-36.

Brennan, Charles D. (1998). Integrating the health care supply chain. En: Health Care Finantial Management, 1998, Vol. 52, No. 1, p. $31-35$.

Capó Vicedo, J.; Tomás Miquel, J. y Expósito-Langa, M. (2007). La gestión del conocimiento en la cadena de suministro: Análisis de la influencia del contexto organizativo. En: Revista Información tecnológica, Volumen 18, No 1, p. 127-136.

Cambra Fierro, J. J. y Polo Redondo, Y. (2008). La cadena de suministro: primer escalón hacia el éxito de la empresa. En: Harvard Deusto business review, No. 172, p.70-79.

CRC -Congreso de la Republica de Colombia- (2015). Ley 1438. Reforma al Sistema General de Seguridad Social en Salud.

Eisenhardt, K. M. (1989). Building Theories from Case Study Research. En: Academy of Management Review, Vol. 14, No 4, p. 532-550.

Franco C.; Flórez A. y Ochoa, M. (2008). Análisis de la cadena de suministros de biocombustibles en Colombia. En: Revista de Dinámica de Sistemas, Vol. 4 No. 2, Pág. 109-133. 
García C. Rafael G.; Torres V. Sergio; Olaya E., Érika S.; Díaz G., Héctor B.; Vallejo D., Bibiana M. y Castro S., Hugo F. (2009).Creación de valor en la cadena de abastecimiento del sector salud en Colombia. En: Cuadernos de Administración, Vol. 22, No. 39, julio-diciembre, p. 235-256.

Garrido, Francisco J. (2001). El análisis de redes en el desarrollo local, p. 49-64. En: Montañés Serrano, Manuel; Rodríguez-Villasante Prieto, Tomás, Gutiérrez, Pedro Martín (coords.). Prácticas locales de creatividad social, Madrid: El Viejo Topo, 214p.

Giannakis, Mihalis; Croom, Simon y Slack, Nigel (2004). Supply chain paradigms. En: Understanding supply chains: Concepts, critiques and futures. Oxford University Press, p. 1-22.

Gillies Robin R.; Shortell Stephen M. y Anderson, David A. (1993). Conceptualizing and measuring integration: Findings from the health systems integration study. Hospital and Health Services Administration, Vol. 38, No. 4, p. 467-489.

Hernández-Rodríguez, C. N. R. (2012). Diseño de un modelo general para la gestión de sistemas logísticos en empresas cubanas: consideraciones teóricas y prácticas. En: Santiago, No 127, enero-abril, Facultades de Ciencias Sociales y Humanidades, Universidad de Oriente, Cuba, p.188-199.

Javalgi, Rajshekhar G. y Reinsenwithz, Timothy H. (2001). International logistics operation of MNCs: An exploration of the pharmaceutical industry. En: The Review of Business Journal, Vol. 22, No. 1/2, p. $43-51$.

Jiménez Sánchez, J. y Hernández García, S. (2002). Marco conceptual de la cadena de suministro: un nuevo enfoque logístico. Instituto Mexicano del Transporte. Publicación técnica, No. 215, 272p.

Jiménez Sánchez, J. (2004). Los factores críticos de éxito de la cadena de suministro. Instituto Mexicano del Transporte. Publicación técnica, No. 237, 189p.

Jordana, Jacint (1995). El análisis de los policy network ¿Una Nueva Perspectiva sobre la relación entre Políticas Públicas y Estado? Barcelona: Universidad de Pompeu Fabra. En: Gestión y Análisis de Políticas Públicas, No. 3, mayo-agosto, p. 505-525.

Kongstvedt, Peter R. y Gates, Ruth (1996). Ten critical success factors for integrated delivery systems. Washington: Jones \& Bartlett Learning, 20p.

Klijn, Erik-Hans. (1998). Redes de políticas públicas: Una visión general. Reimpresión y traducción. Londres: Sage, 45p.

Lau L., Santiago y Soler C., Silvio F. (2009). Las redes integradas de servicios de salud desde la realidad cubana. En: Revista Cubana de Salud Pública, octubre-diciembre, Vol. 35, No. 4, p. $34-43$.

Lin, Blossom; Lin, Yung K. y Lin, Cheng C. (2010). Partnership effectiveness in primary community care networks: A national empirical analysis of partners' coordination infrastructure designs. En: Health Care Management Review, Vol. 35, No. 3, p. 224-234. 
Lozares, Carlos. (1996). La teoría de redes sociales. Papers, No. 48, p. 103-126.

Lozares, Carlos. (2005). Bases socio-metodológicas para el análisis de redes sociales. En: Empiria Revista de Metodología de Ciencias Sociales, No. 10, p. 9-35.

Marsh, David y Rhodes, R.A.W. (1992). Policy Communities and Issue Networks. Beyond Typology, p. 249-287. En: Marsh, D. y Rhodes, R.A.W. (ed.). Policy Networks in British Government. Londres: Routledge, 452p.

Morales Eraso, Natalia (2011). Las redes integradas de servicios de salud: una propuesta en construcción. Universidad del Rosario, Escuela de Medicina y Ciencias de la Salud, Documentos de investigación No. 14, 38p.

MSPSC -Ministerio de Salud y Protección Social de Colombia- (2016). Resolución 429. Por medio de la cual se adopta la Política de Atención Integral en Salud.

Mckone, Kathleen E.; Hamilton, Paul y Willis, Susan B. (2005). The ailing healthcare supply chain: A prescription for change. En: Journal of Supply Chain Management, Vol. 41, No. 1, p. 4-17.

Molina, José Luis (2004). La ciencia de las redes. Apuntes de Ciencia y Tecnología, Vol. 11, No 1, p. 36-42.

Molina, José Luis y Ávila Javier (2009). Talleres de redes sociocéntricas (Ucinet6), redes personales (Egonet) y comparación estadística de grupos (SPSS). 114p.

Montenegro, Hernán; Holder, Reynaldo; Ramagem, Caroline; Urrutia, Soledad y Fabrega, Ricardo. (2011). Combating health care fragmentation through integrated health service delivery networks in the Americas: Lessons learned. En: Journal of Integrated Care, 2011, Vol. 19. No. 5, p. 5-16.

Musso, Pierre (2004). A filosofía da redes, p. 17-38. En: Parente, André. (org.). Tramas da rede: novas dimensões filosóficas, estéticas e políticas da comunicação. Porto Alegre: Sulina, $303 p$

Ocampo-Rodríguez, M. V.; Urrutia, V. F. B.; Montoya-Rojas, J. P. y Bautista-Botton, D. C. (2013). Sistemas y modelos de salud, su incidencia en las redes integradas de servicios de salud. En: Revista Gerencia y Políticas de Salud, Vol.12 No 24, p. 114-129.

OPS -Organización Panamericana de la Salud- (2010). Redes integradas de servicios de salud: Conceptos, opciones de política y hoja de ruta para su implementación en las Américas. Serie: La renovación de la atención primaria de salud en las Américas No. 4. Washington, D.C, $101 \mathrm{p}$

Patton, M.Q. (2002). Qualitative Research and Evaluation Methods. Thousand Oaks, CA: Sage, $832 \mathrm{p}$.

Petrizzo Páez, Mariángela. (2004). Redes e institucionalización: vinculando evidencias empíricas y redes políticas. En: REDES, Revista Hispana para el análisis de redes sociales, Universidad autónoma de Barcelona, Barcelona, p. 7-21. 
Pike, Brigid y Mongan, Deirdre (2014). The integration of health and social care services. Health Research Board, 137p.

Porras Martínez, José Ignacio. (2001). Policy network o redes de política pública: una introducción a su metodología de investigación. En: Estudios Sociológicos, Vol. XIX No. 3, p. 721-745.

Pulgarín Henao, D. M. (2015). Diseño de una red integrada de servicios de salud basada en la gestión de la cadena de suministro: caso de tres organizaciones de salud de la ciudad de Santiago de Cali. Tesis de Maestría, Programa de Logística Integral. Universidad Autonoma de Occidente. Cali, Colombia, 160p.

Quiroga, Águeda. (2003). Introducción al análisis de datos reticulares prácticas con Ucinet 6.0 y NetDrawl versión 1. Departamento de Ciencias Políticas, Universidad Pompeu Fabra, 36p.

Reveco, Carlos y Weber, Richard (2011). Gestión de capacidad en el servicio de urgencia en un hospital público. En: Revista Ingeniería de Sistemas, Vol. XXV, p. 57-75.

Sanchis, Raquel; Poler, Raúl y Ortiz, Ángel (2009).Técnicas para el modelado de procesos de negocio en cadenas de suministro. En: Información Tecnológica, Vol. 20, No. 2, p. 29-40.

Sanz Menéndez, Luis (2003). Análisis de redes sociales: o cómo representar las estructuras sociales subyacentes. En: Apuntes de Ciencia y Tecnología, No. 7, p. 21-29.

Satinsky, Marjorie A. (1998).The foundations of integrated care: facing the challenges of change. Estados Unidos: Jossey-Bass Inc., 292p.

Starfield, Barbara. (1992). Primary care: Concept, evaluation and policy. Oxford University Press, 448p.

Starfield, Barbara. (1998). Primary care: Balancing health needs, services, and technology. Oxford University Press, 452p.

Stadtler, Hartmut y Kilger, Christoph (2005). Supply chain management and advanced planning. Concepts, models, software and case studies. $3^{a}$ edición. Inglaterra: Springer, 512p.

Starfield, B. (2009). General practice as an integral part of the health system. 16th Nordic Conference on General Practice Copenhagen, Denmark. May 13-16.

Suárez, J. A. A.; Acosta, M. I. G.; Joy, T. L.; Urquiaga, A. J. A. y Baez, Y. P. (2015). Modelo de Referencia de Redes de Valor para un desarrollo sostenible. En: Revista de Investigación Agraria y Ambiental (RIAA), Vol. 1, No 2, p. 29-49

Tabarquino Muñoz, Raúl Andrés (2015). La red de política pública de TIC en Colombia. Actores reguladores y principales operadores. En: Revista Estudios Políticos, No. 46, p. 221-241

Toro J., Walter R. (2008). Modelo de simulación prospectiva de la demanda de servicios de salud para enfermedades de alto costo: Aplicación para una entidad promotora de salud colombiana. España: Universidad Politécnica de Valencia. Edición electrónica gratuita.

Vachon, Stephan y Klassen, Robert D. (2002). An exploratory investigation of the effects of supply chain complexity on delivery performance. IEEE Transactions En: Engineering Management, Vol. 49, No. 3, p. 218-230. 
Vásquez N.; María L. y Vargas L., Ingrid. (2006). Redes integradas de servicios de salud: ¿Solución o problema? En: Revista Ciencias de la Salud, Vol. 4, No. 1, p. 5-9.

Velázquez Álvarez, Alejandro y Aguilar Gallegos, Norman. (2005). Manual introductorio al análisis de redes sociales. REDES.

Vickery, Shawnee K.; Jayaram, Jayanth; Droge, Cornelia y Calantone, Roger. (2003). The effects of an integrative supply chain strategy on customer service and financial performance: An analysis of direct versus indirect relationships. En: Journal of Operations Management, Vol. 21, No. 5, p. 523-539.

Mejía Villamizar, Juan Carlos; Palacio León Óscar y Adarme Jaimes Wilson (2013). Efecto Látigo en La Planeación de La Cadena de Abastecimiento, Medición y Control. En: Revista Ciencia e Ingeniería Neogranadina, 2013, Vol. 23, No 2, p. 37-54.

Wasserman, Stanley y Faust, Katherine. (1994). Social Network Analysis: Methods and Applications. Cambridge: Cambridge University, 857p.

Zurbriggen, Cristina. (2011). La utilidad del Análisis de Redes de Políticas Públicas. En: Argumentos, Vol. 24, No. 66, mayo-agosto, Universidad Autónoma Metropolitana, Distrito Federal, México, p. 181-208. 


\section{ANEXOS}

\section{A. GUÍA ENTREVISTA AL GERENTE DE LA RED DE SALUD ORIENTE - EMPRESA SOCIAL DEL ESTADO A PARTIR DE ATRIBUTOS MÍNIMOS DE UNA RISS Y SCOR MODEL}

1. ¿Cuáles son los modelos que dirigen la prestación del servicio de salud las Empresas Sociales del Estado (ESE) de la ciudad Santiago de Cali?

2. ¿Considera usted que existen fragmentaciones dentro de la ESE? ¿Cuáles son? ¿En qué consisten?

3. ¿Cuáles son las herramientas contractuales que legalizan y legitiman el funcionamiento de la ESE Oriente?

4. ¿La ESE Oriente responde a todos los niveles de atención?

5. ¿EL primer nivel de atención de la ESE Oriente es la puerta de entrada al sistema de servicios de salud de la comunidad objetivo?

6. ¿Cuáles son las estrategias de la ESE Oriente para lograr acercamiento hacia la comunidad?

7. ¿Qué planes de sensibilización existen para los usuarios de la ESE Oriente?

8. ¿En qué nivel de desarrollo se encuentra la APS dentro las ESE Oriente?

9. ¿La ESE Oriente trabaja de la mano con otros actores de la comunidad? (escuelas, CAI, estaciones de policía, bomberos, ICBF, etc.).

10. ¿Cómo se controla la idoneidad del personal que presta los servicios de salud y se vincula a la ESE Oriente?

11. ¿Cuáles son los indicadores principales para medir el desempeño de la ESE Oriente?

12. ¿Dentro de los planes de gestión y mejoramiento qué estrategias se plantean para el fortalecimiento de la cadena de abastecimiento de la ESE Oriente?

13. ¿Cuáles considera usted que son las fortalezas y debilidades de la cadena de abastecimiento de las ESE Oriente?

\section{B. GUÍA ENTREVISTA AL EXGERENTE DE LA RED DE SALUD NORTE - EMPRESA SOCIAL DEL ESTADO A PARTIR DE ATRIBUTOS MÍNIMOS DE UNA RISS Y SCOR MODEL}

1. ¿Cuáles son los modelos que dirigen la prestación del servicio de salud las Empresas Sociales del Estado ESE- de la ciudad Santiago de Cali?

2. ¿Las ESE de la ciudad se encuentran en capacidad de atender todos los niveles de atención?

3. ¿Es el primer nivel de atención la puerta de entrada al sistema de servicios de salud?

4. ¿Se encuentran las ESE en capacidad de resolver todos los requerimientos del paciente?

5. ¿Considera usted que existen fragmentaciones dentro de las ESE? 
6. ¿Cuáles son las herramientas contractuales que legalizan y/o legitiman las ESE?

7. ¿Cuáles fueron sus estrategias para lograr acercamiento hacia la comunidad?

8. ¿ ¿Qué planes de sensibilización conoce usted para los usuarios?

9. ¿En qué nivel de desarrollo se encontraba la APS dentro las ESE?

10. ¿La ESE que usted dirigió trabajaba de la mano con otros actores de la comunidad? (escuelas, CAI, estaciones de policía, bomberos, ICBF).

11. ¿Cuáles son las medidas de control que permiten tener personal idóneo para la prestación de servicios de salud?

12. ¿Cómo funciona la contratación del personal que presta los servicios de salud dentro de las ESE?

13. ¿Cuáles son los indicadores principales para medir el desempeño de la ESE?

14. ¿Dentro de los planes de gestión y mejoramiento que estrategias se plantean para el fortalecimiento de la cadena de abastecimiento de las ESE?

15. ¿Cuáles considera usted que son las fortalezas y debilidades de la cadena de abastecimiento de las ESE?

\section{GUÍA DE SOLICITUDES AL EQUIPO DE TRABAJO DE LA ESE ORIENTE A PARTIR DE ATRIBUTOS MÍNIMOS DE UNA RISS Y SCOR MODEL}

\section{Eje de Planear}

1. ¿Cómo funciona la cadena de suministro en la ESE Oriente? ¿Existe un diagrama?

2. ¿Existe rotación de proveedores en la ESE Oriente?

3. ¿Cómo se planea el aprovisionamiento de medicamentos, insumos y servicios requeridos para el desarrollo de la actividad? -Políticas de planeación-

4. ¿Se ha establecido algún patrón de comportamiento de la demanda día - semana - mes - trimestre - semestre - anual?

5. ¿Cómo planifican los presupuestos?

6. ¿Dentro de los planes de gestión y mejoramiento que estrategias se plantean para el fortalecimiento de la cadena de abastecimiento de la ESE Oriente?

7. ¿Cuáles considera Ud. que son las fortalezas y debilidades de la cadena de abastecimiento de la ESE Oriente?

8. ¿Cuáles son los indicadores clave de desempeño (KPI) que maneja?

\section{Eje de Procurar}

9. En el plan de desarrollo 2012-2015 se mencionan fallas con los proveedores de energía, agua y telecomunicaciones. ¿A qué tipo de fallas se refiere y que planes de acción se han implementado para minimizar su impacto en la prestación de servicio? 
10. ¿Cuáles son los servicios de laboratorio que mayor demanda tienen en la ESE Oriente?

11. ¿Cuáles son los medicamentos que mayor demanda tienen en la ESE Oriente?

12. ¿Cuál es el procedimiento para manejar los residuos? ¿En qué porcentaje de cumplimiento se encuentran?

13. ¿Cuáles son los requerimientos que deben cumplir las entidades que desean ser proveedores de la ESE Oriente?

14. ¿Existen evaluaciones a sus proveedores? ¿Cuáles son los criterios que miden en dichas evaluaciones?

15. ¿Cuáles son los indicadores clave de desempeño (KPI) que maneja?

\section{Eje de Distribución}

16. ¿Existen políticas de inventario?

17. ¿Cómo se controla el inventario?

18. ¿Cuál es la rotación de Inventario?

19. ¿Cómo se organiza la distribución de materiales e insumos a los diferentes puntos que conforman la ESE Oriente?

20. ¿Se manejan sistemas de almacenamiento? ¿Cuáles?

21. ¿Cuáles son los indicadores clave de desempeño (KPI) que maneja?

\section{Eje de Costos}

22. ¿Cuáles son los insumos más representativos en materia de costos de la ESE Oriente?

23. ¿Cuáles son los servicios de laboratorio más representativos en materia de costos de la ESE Oriente?

24. ¿Qué porcentaje de los costos totales representa el servicio de urgencias? Dentro de este servicio ¿qué rubros son los más representativos?

25. ¿Qué porcentaje de los costos totales representa el servicio de salud oral? Dentro de este servicio ¿qué rubros son los más representativos?

26. ¿Qué porcentaje de los costos totales representa la papelería administrativa? Dentro de este costo ¿cuáles insumos son los más representativos?

\section{Eje de Comunidad}

27. Actualmente ¿̇cuál es la demanda de servicios de salud que maneja esta comunidad?

28. ¿Considera usted que están en capacidad de satisfacer esta demanda?

29. ¿Cuáles son los servicios de salud que mayor participación tienen en dicha demanda? 
30. ¿Cuáles han sido las estrategias para lograr acercamiento hacia la comunidad?

31. ¿Existen planes de sensibilización a los usuarios?

32. ¿En qué nivel de desarrollo se encuentra la APS dentro la ESE Oriente?

33. ¿La ESE Oriente trabaja de la mano con otros actores de la comunidad? (escuelas - CAI estaciones de policía - bomberos - ICBF)

\section{Eje de Recurso Humano}

34. ¿Cómo funciona la programación del personal médico de la ESE Oriente?

35. ¿Cuáles son los requerimientos para la selección del personal que se vincula a la red?

36. ¿Existen evaluaciones del personal que actualmente labora en la ESE Oriente?

37. ¿Qué acciones correctivas tienen implementadas en caso de alguna falla del personal?

\section{Eje de Control Interno}

38. ¿Han logrado la implementación del modelo MECI (Modelo estándar de control interno)? ¿Cómo ha sido la experiencia de la implementación?

39. ¿Cuál otro modelo aplica para autorregularse?

40. ¿ ¿Se efectúa seguimiento al plan de acción de cada año? ¿Cuáles han sido los resultados?

\section{Eje de Sistemas de Información}

41. ¿La ESE Oriente cuenta con información actualizada y real sobre los usuarios que atiende actualmente?

42. ¿Qué sistema de información en red utilizan para dicho control?

43. ¿El software que utiliza la ESE Oriente permite visualizar estadísticas y gráficos para su respectivo análisis

44. ¿Se encuentran vinculadas todas las entidades de la ESE Oriente a dicho sistema de información?

45. ¿Cuáles son las estrategias que se implementan para que la información fluya correctamente en la ESE Oriente? ¿Estas herramientas han sido eficaces?

\section{Eje de Calidad}

46. Cantidad de requerimientos atendidos en el año 2012-2013

47. ¿Cuál es la capacidad instalada de la ESE Oriente?

48. ¿Existen convenios interinstitucionales para prestar servicios de salud?

49. Cantidad de consultas ambulatorias 2012-2013

50. Mortalidad Materna 
51. Promedio día estancia

52. Tasa mortalidad servicio urgencia

53. Numero consulta urgencia general

54. Número de consulta urgencia especialista

\section{SALIDAS DE MATRICES DE UCINET 6.0}

Matriz de la cadena de suministro de la ESE Oriente de Cali, sin la aplicación del concepto de RISS

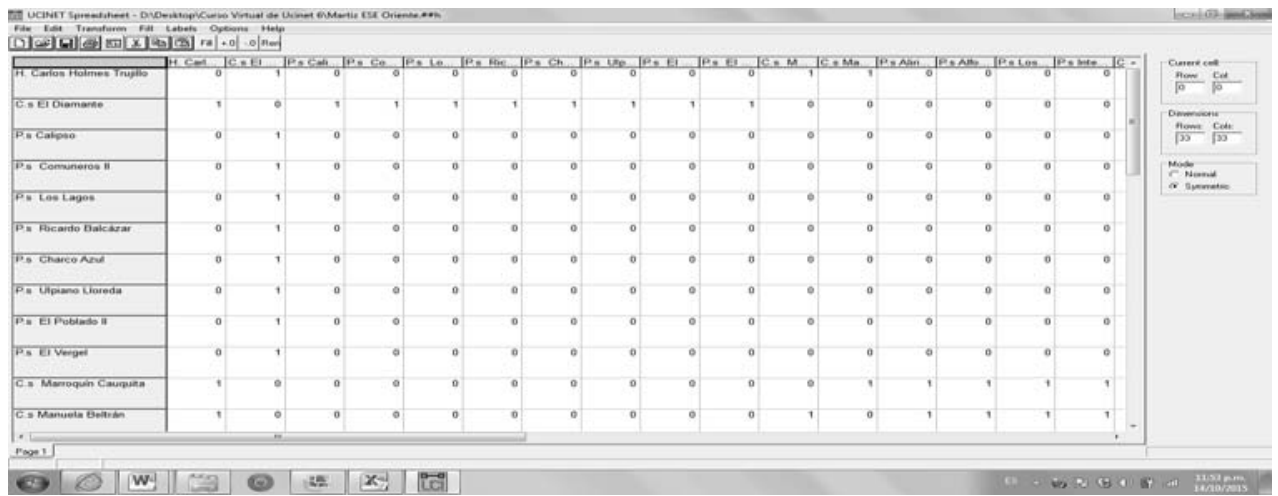

Fuente: Elaboración propia con Ucinet 6.0

Matriz de la cadena de suministro con la aplicación del concepto de la RISS

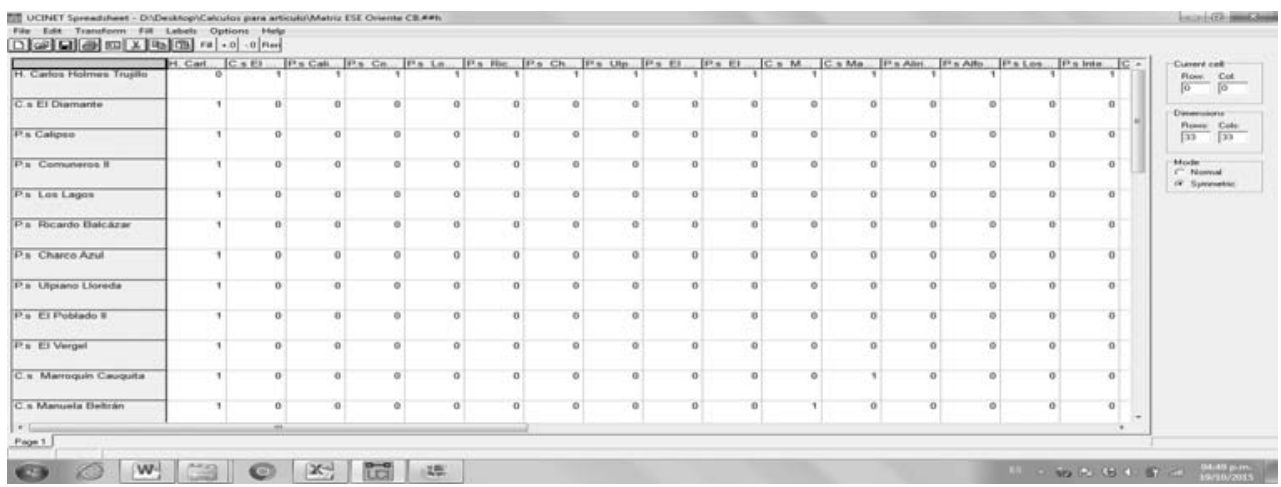

Fuente: Elaboración propia con Ucinet 6.0 
Dora Marcela Pulgarín Henao - Raúl Andrés Tabarquino Muñoz

Matriz de actores de la ESE Oriente con los 14 atributos de la RISS

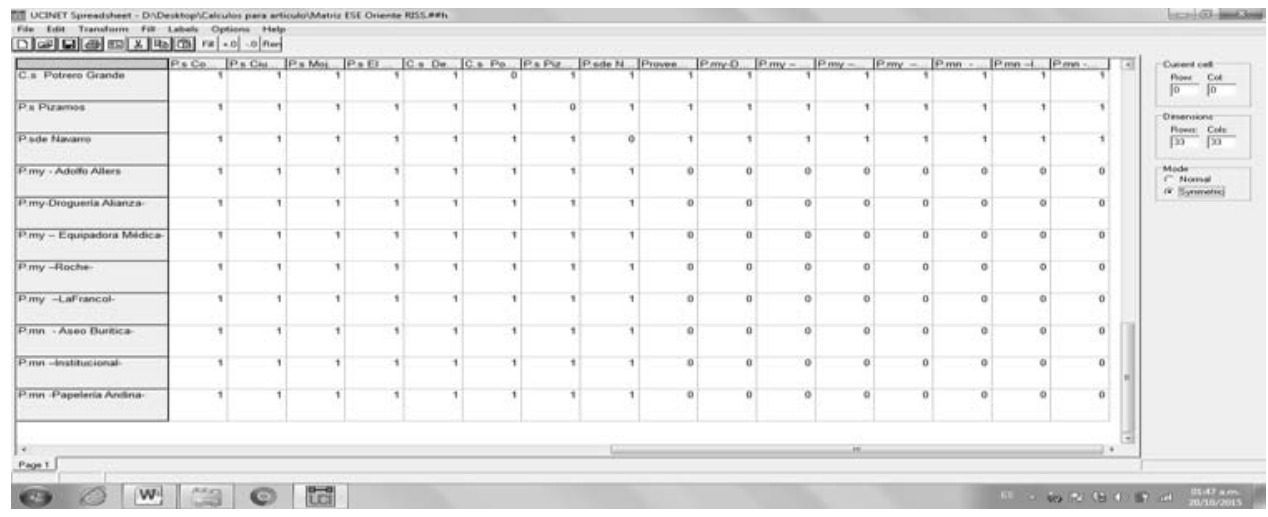

Fuente: Elaboración propia con Ucinet 6.0 